\title{
CFT Correlators for Cardy Bulk Fields via String-Net Models
}

\author{
Christoph SCHWEIGERT and Yang YANG
}

Fachbereich Mathematik, Universität Hamburg, Bereich Algebra und Zahlentheorie, Bundesstraße 55, 20146 Hamburg, Germany

E-mail: christoph.schweigert@uni-hamburg.de, Yang.Yang@studium.uni-hamburg.de

URL: https://www.math.uni-hamburg.de/home/schweigert/

Received November 01, 2020, in final form April 12, 2021; Published online April 21, 2021

https://doi.org/10.3842/SIGMA.2021.040

\begin{abstract}
We show that string-net models provide a novel geometric method to construct invariants of mapping class group actions. Concretely, we consider string-net models for a modular tensor category $\mathcal{C}$. We show that the datum of a specific commutative symmetric Frobenius algebra in the Drinfeld center $Z(\mathcal{C})$ gives rise to invariant string-nets. The Frobenius algebra has the interpretation of the algebra of bulk fields of the conformal field theory in the Cardy case.
\end{abstract}

Key words: two-dimensional conformal field theory; string-net models; correlators; Cardy case

2020 Mathematics Subject Classification: $81 \mathrm{~T} 40$

\section{Introduction}

Two-dimensional conformal field theories, to which we refer as a CFT in the following, are quantum field theories that apart from their intrinsic physical interest, are amenable to a precise mathematical study. In this paper, we use string-net models to study consistent systems of bulk field correlators in a class of such models.

A consistent system of correlators in a CFT is obtained by specifying elements in spaces of conformal blocks, subject to certain consistency conditions. For a conformal field theory with the monodromy data given by a braided monoidal category $\mathcal{D}$, the spaces of conformal blocks can be constructed as morphism spaces in $\mathcal{D}$. They are endowed with projective actions of mapping class groups given in terms of the structures on $\mathcal{D}$. For a rational conformal field theory, the category $\mathcal{D}$ is a (semisimple) modular tensor category and the spaces of conformal blocks are provided by the state spaces of a three-dimensional topological field theory, namely the Reshetikhin-Turaev TFT based on $\mathcal{D}$. In this framework, the task of finding a consistent system of correlators is equivalent to finding for each surface $\Sigma$ a vector in the space of conformal blocks on the double $\widehat{\Sigma}$. This element has to be invariant under the action of the mapping class group of $\Sigma$ and the set of elements has to be consistent under sewing of the surfaces. This problem has been solved completely in $[7,10,11,12,13]$, using in a non-trivial way the geometry of certain 3-manifolds. This is not only technically involved, but also a serious obstacle to extend the approach to more general classes of CFTs, e.g., those based on non-semisimple modular tensor categories, since a 3d-TFT of Reshetikhin-Turaev type with values in vector spaces can only be constructed for semisimple MTCs.

In this article, we only consider bulk fields on oriented surfaces. Instead of considering the double $\widehat{\Sigma}$ of the surface $\Sigma$, which for $\Sigma$ oriented without boundary consists of two copies of $\Sigma$ 
with opposite orientation, i.e., $\hat{\Sigma}=\Sigma \sqcup \bar{\Sigma}$ (see, e.g., [10, Section 5.1]), one uses the following relation for the state space of the Reshetikhin-Turaev TFT:

$$
Z_{\mathrm{RT}, \mathcal{C}}(\hat{\Sigma})=Z_{\mathrm{RT}, \mathcal{C}}(\Sigma \sqcup \bar{\Sigma}) \cong Z_{\mathrm{RT}, \mathcal{C}}(\Sigma) \otimes Z_{\mathrm{RT}, \mathcal{C}}(\bar{\Sigma}) \cong Z_{\mathrm{RT}, \mathcal{C}^{\mathrm{rev}} \otimes \mathcal{C}}(\Sigma)
$$

and can take the enveloping category $\mathcal{C}^{\text {rev }} \otimes \mathcal{C}$ of a modular tensor category as the category $\mathcal{D}$ and stick with the original surface $\Sigma$. Modularity implies that we have a braided equivalence: $\mathcal{C}^{\text {rev }} \otimes \mathcal{C} \simeq Z(\mathcal{C})$, where $Z(\mathcal{C})$ is the Drinfeld center of $\mathcal{C}$, see, e.g., [24] for a statement that includes non-semisimple categories as well. It is shown in $[3,4,18,26]$ that the Reshetikhin-Turaev construction for $Z(\mathcal{C})$ is equivalent to the extended Turaev-Viro-Barrett-Westbury state-sum construction based on $\mathcal{C}$, hence we have

$$
Z_{\mathrm{RT}, \mathcal{C}^{\mathrm{rev}} \otimes \mathcal{C}}(\Sigma) \cong Z_{\mathrm{RT}, Z(\mathcal{C})}(\Sigma) \cong Z_{\mathrm{TV}, \mathcal{C}}(\Sigma)
$$

The string-net model was first introduced in the study of topological order in condensed matter physics by Levin and Wen [22]. The collection of state spaces associated to surfaces are described by equivalence classes of string-diagrams on compact oriented surfaces with boundaries and can be extended to a once-extended TFT which has recently been shown to be equivalent to the Turaev-Viro-Barrett-Westbury state-sum construction [15, 17]. The string-net model has two advantages that are attractive in our context: first, a vector in the space of conformal blocks can be described by a string-net, and second, the action of the mapping class group, when expressed in terms of such vectors, is completely geometrical (in fact, the consideration of mapping class groups actions on string-nets has already appeared in [19]).

In this paper, we first define fundamental string-nets on a generating set of surfaces for every commutative symmetric Frobenius algebra $F$ in the Drinfeld center $Z(\mathcal{C})$, using the structure morphisms of $F$. We show in Lemma 3.8 that those string-nets are invariant under the mapping class group action. Moreover, the prescription extends to a consistent system of correlators in the sense of [14] by sewing, where the Frobenius algebra $F$ befits the algebra of bulk fields, provided that the string-net on the torus with one boundary circle is invariant under the mapping class group action. It can be inferred from the known result [21, Theorem 3.4] that a haploid commutative symmetric Frobenius algebra $F \in Z(\mathcal{C})$ satisfies this condition if and only if $\operatorname{dim}(F)$ equals the global dimension of the category $\mathcal{C}$. Then to each surface $\Sigma$, possibly with non-empty boundary, the assigned correlator can be obtained as a string-net by decomposing the surface into pairs of pants and placing the appropriate fundamental string-nets on each component. For instance, for a surface of genus one with one ingoing and two outgoing boundary components, we have the following string-net

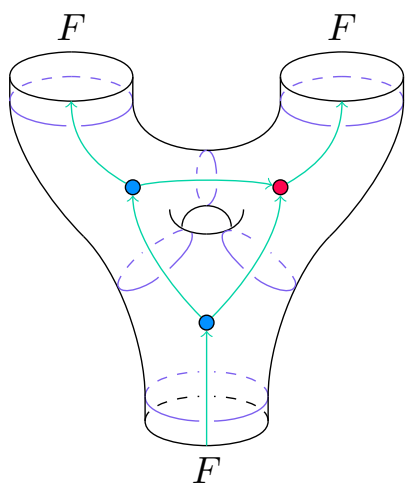

Figure 1. The string-net assigned to the extended surface of genus one with one ingoing and two outgoing boundary circles according to a certain pairs of pants decomposition. 
Here the green lines are labeled by the Frobenius algebra $F$, the red and blue circular coupons stand for the multiplication and co-multiplication of the Frobenius algebra $F$ respectively, while the purple circles stand for the boundary projectors (introduced in Remark 2.6) that account for the half-braiding of $F$.

We then restrict to a specific algebra $F_{1}$ satisfying the condition of modular invariance: the bulk algebra for the Cardy case in which the modular invariant on a torus is given by the charge conjugation matrix. The underlying object of the algebra $F_{1}$ is

$$
L=\bigoplus_{i \in \mathcal{I}(\mathcal{C})} X_{i}^{\vee} \otimes X_{i} \in \mathcal{C}
$$

along with a certain half-braiding (see Section 4.1). Here $\mathcal{I}(\mathcal{C})$ stands for the set of isomorphism classes of simple objects in $\mathcal{C}$ and $X_{i}$ is a fixed representative for each $i \in \mathcal{I}(\mathcal{C})$. We show that for the algebra $F_{1}$, the string-nets describing the correlators are almost empty (Theorem 4.5). For instance, the string-net shown in Figure 1, after substituting the algebra with $F_{1}$, will be shown to be the following string-net

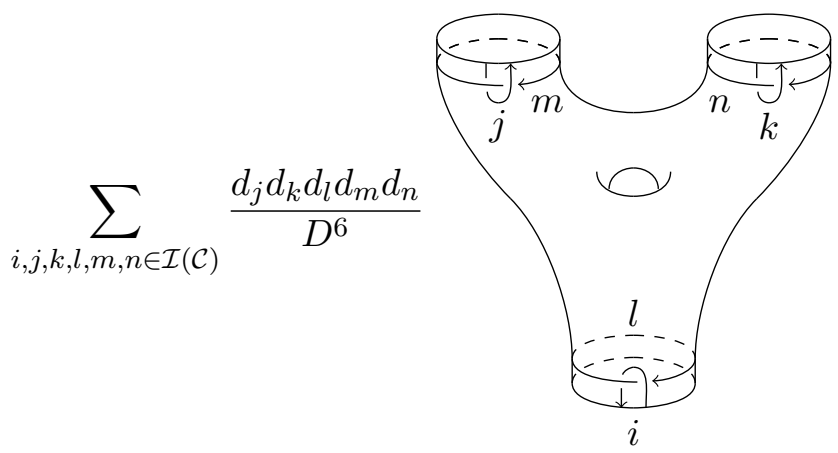

Figure 2. The simplified form of the string-net assigned to the extended surface $\Sigma_{1 \mid 2}^{1}$.

These correlators have been constructed in terms of the evaluation of a $3 \mathrm{~d}$-TFT on certain ribbon graphs in 3-manifolds in [6]. The geometry and the ribbon graphs are quite involved. The fact that we can describe them by almost empty string-nets demonstrates the advantage of the string-net construction.

This paper is organized as follows: in Section 2, we briefly review string-net models, following [17]. We next recall some facts about modular tensor categories in Section 3.1, review the notion of a consistent system of bulk field correlators in Section 3.2. We define the fundamental string-nets in Section 3.3 and show that they give rise to a consistent system of correlators when the used Frobenius algebra is modular. Section 4 is devoted to the Cardy case.

We expect that our results can be generalized in several directions: beyond the Cardy case and to correlators including also boundary and defect fields. A generalization of the string net construction to non-semisimple finite tensor categories remains, at the moment, a challenge. It would allow us to address correlators of logarithmic conformal field theories as well in a twodimensional setting.

\section{String-net models}

\subsection{Spherical fusion categories}

String-net models are defined for spherical fusion categories. In this section, we review some basic facts of spherical fusion categories and fix our notations. We denote by $\mathbb{K}$ an algebraically closed field of characteristic 0 . 
Recall that a right dual of an object $V$ in a strict monoidal category $\mathcal{C}$ is an object $V^{\vee}$ together with morphisms $\operatorname{coev}_{V} \in \operatorname{Hom}_{\mathcal{C}}\left(\mathbb{I}, V \otimes V^{\vee}\right)$ and $\operatorname{ev}_{V} \in \operatorname{Hom}_{\mathcal{C}}\left(V^{\vee} \otimes V, \mathbb{I}\right)$ satisfying

$$
\left(\mathrm{id}_{V} \otimes \mathrm{ev}_{V}\right) \circ\left(\operatorname{coev}_{V} \otimes \mathrm{id}_{V}\right)=\mathrm{id}_{V}
$$

and

$$
\left(\mathrm{ev}_{V} \otimes \mathrm{id}_{V^{\vee}}\right) \circ\left(\mathrm{id}_{V^{\vee}} \otimes \operatorname{coev}_{V}\right)=\mathrm{id}_{V^{\vee}}
$$

We depict the right duality maps graphically as
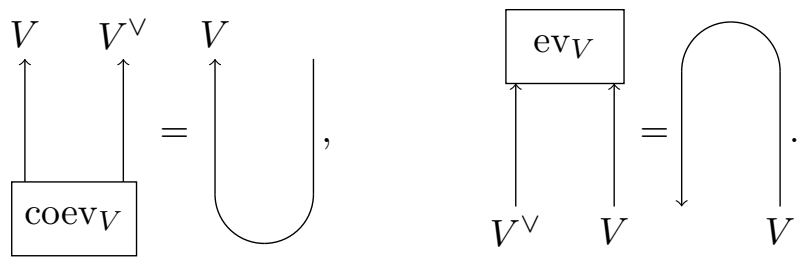

Here we replaced $V^{\vee}$ by $V$ upon reversing the direction of the arrow. Left duality is defined similarly by reversing the arrows in the graphical notation. A monoidal category in which every object has both left and right duals is called a rigid monoidal category.

A pivotal structure on a rigid monoidal category is a monoidal natural isomorphism $\omega: \operatorname{id}_{\mathcal{C}} \Rightarrow(-)^{\vee \vee}$. A pivotal structure is called strict if $\operatorname{id}_{\mathcal{C}}=(-)^{\vee \vee}$ and $\omega=\mathrm{id}_{\mathrm{id}_{\mathcal{C}}}$. It is known that every pivotal category is pivotally equivalent to a pivotal category with strict pivotal structure [23, Theorem 2.2], hence we will assume the pivotal structure to be strict in the following without loss of generality. For a strict pivotal category, the left and right duality strictly coincide as functors.

In a pivotal category we have the notions of right and left traces for any $f \in \operatorname{End}_{\mathcal{C}}(V)$. Graphically

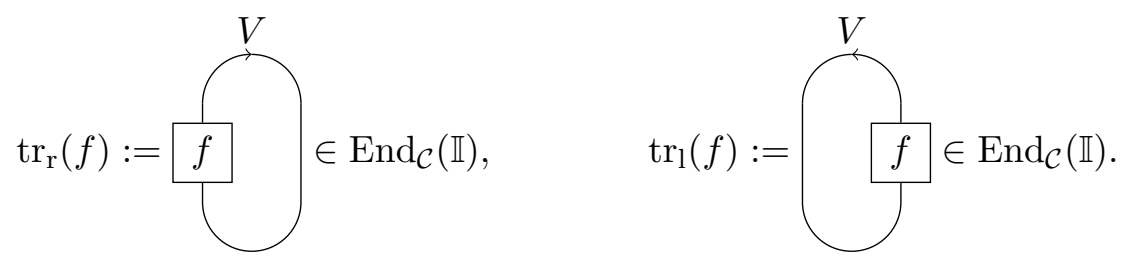

When applied to $\operatorname{id}_{V} \in \operatorname{End}_{\mathcal{C}}(V)$, we get the definitions of the left and right categorical dimension of the object $V \in \mathcal{C}$. A pivotal category is called spherical if the left and right traces coincide, i.e., $\operatorname{tr}(f):=\operatorname{tr}_{\mathrm{r}}(f)=\operatorname{tr}_{l}(f)$ and $\operatorname{dim}(V):=\operatorname{dim}_{\mathrm{r}}(V)=\operatorname{dim}_{l}(V)$.

Definition 2.1. A fusion category over $\mathbb{K}$ is a rigid $\mathbb{K}$-linear monoidal category $\mathcal{C}$ that is finitely semisimple, with the monoidal unit $\mathbb{I}$ being simple. A spherical fusion category over $\mathbb{K}$ is a spherical category $\mathcal{C}$ that is also a fusion category over $\mathbb{K}$.

Here being $\mathbb{K}$-linear means that the sets of morphisms are $\mathbb{K}$-vector spaces and the composition as well as the monoidal product are bilinear. Being finitely semisimple means that there are finitely many isomorphism classes of simple objects (objects with no non-trivial subobject) and every object is a direct sum of finitely many simple objects. Note that $\mathbb{K}$ linearity and finite-semisimplicity together imply that the morphism spaces are finite dimensional.

Let us denote the set of isomorphism classes of simple objects by $\mathcal{I}(\mathcal{C})$, and fix a representative $X_{i}$ for each $i \in \mathcal{I}(\mathcal{C})$. In addition, we require $0 \in \mathcal{I}(\mathcal{C})$ and $X_{0}=\mathbb{I}$. Duality furnishes a involution on $\mathcal{I}(\mathcal{C})$, i.e., $i \mapsto \bar{i}:=\left[X_{i}^{\vee}\right]$. We require that $X_{\bar{i}}=X_{i}^{\vee}$ whenever $i \neq \bar{i}$. Since $\mathbb{K}$ is assumed to be algebraically closed, the only finite dimensional division algebra over $\mathbb{K}$ is $\mathbb{K}$ itself. Thus 
we have Schur's lemma: $\operatorname{Hom}_{\mathcal{C}}\left(X_{i}, X_{j}\right) \cong \delta_{i, j} \mathbb{K}$. In particular, $d_{X}:=\operatorname{dim}(X) \in \operatorname{End}_{\mathcal{C}}(\mathbb{I}) \cong \mathbb{K}$. Define the global dimension of the spherical fusion category $\mathcal{C}$ to be

$$
D^{2}:=\sum_{i \in \mathcal{I}(\mathcal{C})} d_{i}^{2}
$$

Despite of the notation, we do not choose a square root of the global dimension. By [5, Theorem 2.3], $D^{2} \neq 0$.

We define the functor $\underbrace{\mathcal{C} \otimes \cdots \otimes \mathcal{C}}_{n} \rightarrow \mathcal{V}$ ect $\mathbb{K}_{\mathbb{K}}$ by

$$
V_{1} \otimes \cdots \otimes V_{n} \mapsto \operatorname{Hom}_{\mathcal{C}}\left(\mathbb{I}, V_{1} \otimes \cdots \otimes V_{n}\right) .
$$

The pivotal structure furnishes a natural isomorphism by

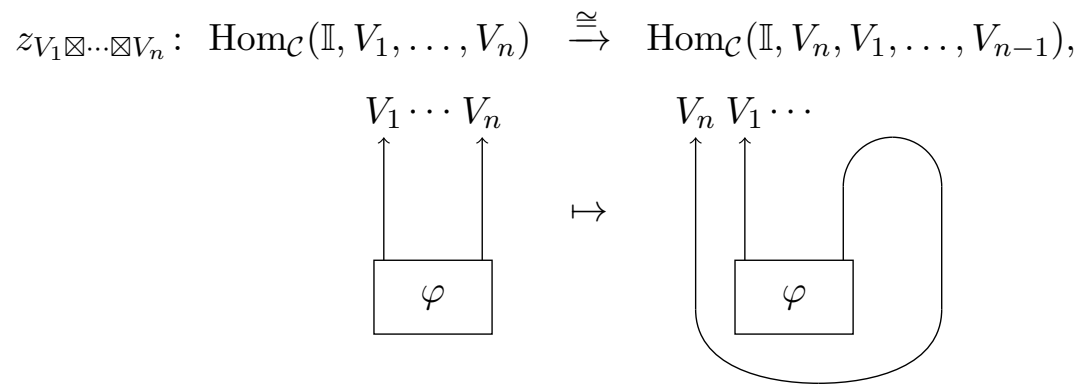

It can be seen that $z^{n}=\mathrm{id}$. Thus, up to a natural isomorphism, $\operatorname{Hom}_{\mathcal{C}}\left(\mathbb{I}, V_{1}, \ldots, V_{n}\right)$ depends only on the cyclic order of $V_{1}, \ldots, V_{n}$. This allows us to represent an element $\varphi \in$ $\operatorname{Hom}_{\mathcal{C}}\left(\mathbb{I}, V_{1}, \ldots, V_{n}\right)$ by a round coupon with $n$ outgoing legs colored by $V_{1}, \ldots, V_{n}$ in clockwise order

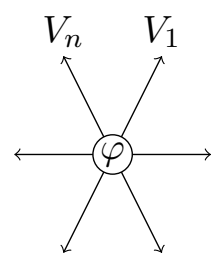

We are able to connect legs with dual labels: define the composition map

$$
\begin{aligned}
\operatorname{Hom}_{\mathcal{C}}\left(\mathbb{I}, V_{1}, \ldots, V_{n}, X^{\vee}\right) \otimes_{\mathbb{K}} \operatorname{Hom}_{\mathcal{C}}\left(\mathbb{I}, X, W_{1}, \ldots, W_{m}\right) & \rightarrow \operatorname{Hom}_{\mathcal{C}}\left(\mathbb{I}, V_{1}, \ldots, V_{n}, W_{1}, \ldots, W_{m}\right), \\
\varphi \otimes_{\mathbb{K}} \psi & \mapsto \varphi \circ_{X} \psi:=\operatorname{ev}_{X} \circ\left(\varphi \otimes_{\mathbb{K}} \psi\right) .
\end{aligned}
$$

This gives rise to a pairing: $\operatorname{Hom}_{\mathcal{C}}\left(\mathbb{I}, V_{1}, \ldots, V_{n}\right) \otimes_{\mathbb{K}} \operatorname{Hom}_{\mathcal{C}}\left(\mathbb{I}, V_{n}^{\vee}, \ldots, V_{1}^{\vee}\right) \rightarrow \mathbb{K}$. It is nondegenerate due to the nondegeneracy of the evaluation maps. Hence for any choice of bases $\left\{\varphi_{\alpha}\right\}_{\alpha \in A}$ of $\operatorname{Hom}_{\mathcal{C}}\left(\mathbb{I}, V_{1}, \ldots, V_{n}\right)$, we define the dual bases $\left\{\varphi^{\alpha}\right\}_{\alpha \in A}$ with respect to this nondegenerate pairing. In the following we will use the following summation convention

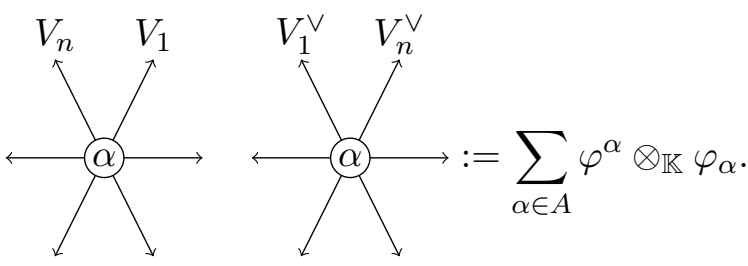

Such expressions are independent of the choice of bases.

We now introduce the following useful completeness relation: 
Proposition 2.2. For any $V_{1}, \ldots, V_{n} \in \mathcal{C}$, we have

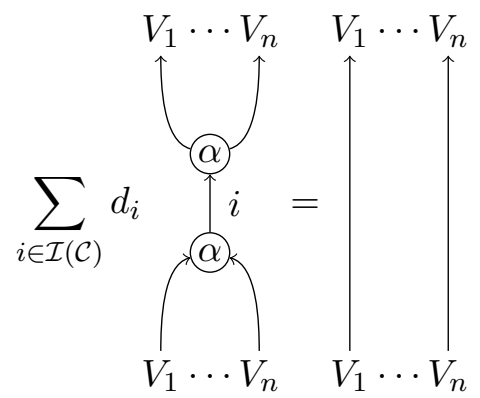

\subsection{The string-net construction}

We now give a brief introduction to the string-net construction. We refer to [17] for more details, and to [22] for motivations from physics.

Let's consider finite graphs (i.e., the sets of the vertices and the edges are both finite) embedded in an oriented surface $\Sigma$, which is not required to be compact and may have non-empty boundary. For such a graph $\Gamma$, denote by $\mathcal{E}^{\text {or }}(\Gamma)$ the set of its oriented edges and $\mathcal{V}(\Gamma)$ the set of its vertices. One-valent vertices are called endings. We denote the set of endings of $\Gamma$ by $\mathcal{V}^{\text {en }}(\Gamma)$, and define $\mathcal{V}^{\text {in }}(\Gamma):=\mathcal{V}(\Gamma) \backslash \mathcal{V}^{\text {en }}(\Gamma)$. We require $\Gamma \cap \partial \Sigma=\mathcal{V}^{\text {en }}(\Gamma)$. We will call the edges terminating at endings legs. Note that we don't make a choice of orientations for the edges of the finite graphs.

Definition 2.3. Let $\mathcal{C}$ be a spherical fusion category, $\Sigma$ and $\Gamma$ be as defined above. A $\mathcal{C}$-coloring (or simply coloring when there is no ambiguity) of $\Gamma$ is given by the following data:

- A map $V: \mathcal{E}^{\text {or }}(\Gamma) \rightarrow \operatorname{Obj}(\mathcal{C})$ such that for every $e \in \mathcal{E}^{\text {or }}(\Gamma)$, we have $V(e)=V(\bar{e})^{*}$, where $\bar{e}$ is the edge with opposite orientation of $e$.

- A choice of a vector $\varphi(v) \in \operatorname{Hom}_{\mathcal{C}}\left(\mathbb{I}, V\left(e_{1}\right), \ldots, V\left(e_{n}\right)\right)$ for every $v \in \mathcal{V}^{\text {in }}(\Gamma)$, where $e_{1}, \ldots, e_{n}$ are incident to $v$, taken in clockwise order (when the orientation of the surface is considered conterclockwise) and with outward orientation.

An isomorphism $f$ of two colorings $(V, \varphi)$ and $\left(V^{\prime}, \varphi^{\prime}\right)$ is a collection of isomorphisms $f_{e}: V(e) \cong$ $V^{\prime}(e)$ that is compatible with $V(e)=V(\bar{e})^{*}$ and such that $\varphi^{\prime}(v)=\left(\bigotimes_{e \in \mathcal{E}^{\circ \mathrm{r}}(v)} f_{e}\right) \circ \varphi(v)$, where $\mathcal{E}^{\text {or }}(v)$ is the set of edges that are incident to the vertex $v$.

Let $B \subset \partial \Sigma$ be a finite collection of points on $\partial \Sigma$ and $\mathbf{V}: B \rightarrow \operatorname{Obj}(\mathcal{C})$ a map. A $\mathcal{C}$-colored graph $\Gamma$ with boundary value $\mathbf{V}$ is a colored graph such that $\mathcal{V}^{\mathrm{en}}(\Gamma)=B$ and $V\left(e_{b}\right)=\mathbf{V}(b)$, where $b \in B$ and $e_{b}$ is the edge incident to $b$ with outgoing orientation. We define $\operatorname{Graph}(\Sigma, \mathbf{V})$ to be the set of $\mathcal{C}$-colored graphs in $\Sigma$ with boundary value $\mathbf{V}$, and $\operatorname{VGraph}(\Sigma, \mathbf{V})$ to be the $\mathbb{K}$-vector space freely generated by $\operatorname{Graph}(\Sigma, \mathbf{V})$.

When $\Sigma$ happens to be a disc $D \subset \mathbb{R}^{2}$, a colored graph $\Gamma \in \operatorname{Graph}(D, \mathbf{V})$ can be naturally viewed as the graphical representation of some morphism in $\mathcal{C}$. Indeed, graphical calculus for spherical fusion categories provides a canonical linear surjection [17, Theorem 2.3]

$$
\langle-\rangle_{D}: \operatorname{VGraph}(\Sigma, \mathbf{V}) \rightarrow \operatorname{Hom}_{\mathcal{C}}\left(\mathbb{I}, V\left(e_{1}\right), \ldots, V\left(e_{n}\right)\right),
$$

where $B=\left\{b_{1}, \ldots, b_{n}\right\}$ and $e_{1}, \ldots, e_{n}$ are the corresponding outgoing legs, taken in the clockwise order.

The finite dimensional vector $\operatorname{space} \operatorname{Hom}_{\mathcal{C}}\left(\mathbb{I}, V\left(e_{1}\right), \ldots, V\left(e_{n}\right)\right) \cong \operatorname{VGraph}(D, \mathbf{V}) / \operatorname{ker}\langle-\rangle_{D}$ can be viewed as the space of linear combinations of $\mathcal{C}$-colored graphs with a fixed boundary value, where two combinations are identified if they represent the same morphism in $\mathcal{C}$ according 
to the graphical calculus. The identification in turn allows us to perform graphical calculus in this space. This inspires us to use $\operatorname{VGraph}(D, \mathbf{V}) / \operatorname{ker}\langle-\rangle_{D}$ as a local model to define a vector space for an arbitrary oriented surface $\Sigma$ with a prescribed boundary value $\mathbf{V}$, so that we can perform graphical calculus locally.

Definition 2.4. Let $D \subset \Sigma$ be an embedded disc. A null graph with respect to $D$ is a linear combination of colored graphs $\boldsymbol{\Gamma}=c_{1} \Gamma_{1}+\cdots+c_{n} \Gamma_{n} \in \operatorname{VGraph}(\Sigma, \mathbf{V})$ such that

- $\boldsymbol{\Gamma}$ is transversal to $\partial D$ (i.e., no vertex of $\Gamma_{i}$ is on $\partial D$ and the edges of each $\Gamma_{i}$ intersect with $\partial D$ transversally).

- All $\Gamma_{i}$ coincide outside of $D$.

- $\langle\boldsymbol{\Gamma}\rangle_{D}=\sum_{i} c_{i}\left\langle\Gamma_{i} \cap D\right\rangle_{D}=0$.

Denote by $\mathrm{N}(\Sigma, \mathbf{V}) \subset \operatorname{VGraph}(\Sigma, \mathbf{V})$ the subspace spanned by null graphs for all possible embedded disks $D \subset \Sigma$.

Definition 2.5. Let $\Sigma$ be an oriented surface and let $\mathbf{V}: B \rightarrow \operatorname{Obj}(\mathcal{C})$ be a boundary value. Define the string-net space for $(\Sigma, \mathbf{V})$ to be the quotient space

$$
Z_{\mathrm{SN}, \mathcal{C}}(\Sigma, \mathbf{V}):=\operatorname{VGraph}(\Sigma, \mathbf{V}) / \mathrm{N}(\Sigma, \mathbf{V}) \text {. }
$$

As before, we have a linear surjection

$$
\langle-\rangle_{\Sigma}: \operatorname{VGraph}(\Sigma, \mathbf{V}) \rightarrow Z_{\mathrm{SN}, \mathcal{C}}(\Sigma, \mathbf{V})
$$

The map has several nice properties. For instance, it is linear in the colors of vertices and additive with respect to direct sums, isotopic graphs and graphs with isomorphic colorings have the same image. But most importantly, it allows us to identify graphs that only differ by local relations that are encoded by the definition of null graphs. That is to say, all equations from the graphical calculus for the spherical fusion category $\mathcal{C}$, e.g., the one from Proposition 2.2, holds true inside any embedded disc on the surface.

\subsection{Drinfeld center and the extended string-net spaces}

One can associate to any monoidal category $\mathcal{C}$ a braided monoidal category $Z(\mathcal{C})$, called the Drinfeld center of $\mathcal{C}$. Recall that the objects of the Drinfeld center $Z(\mathcal{C})$ are given by the pairs $Y=\left(\dot{Y}, \gamma_{Y}\right)$, where $\dot{Y} \in \mathcal{C}$ and $\gamma_{Y}: \dot{Y} \otimes-\Rightarrow-\otimes \dot{Y}$ is a natural isomorphism called the halfbraiding subjected to certain conditions. We use the over-crossing of a green line labeled by an object $Y \in Z(\mathcal{C})$ to denote its half-braiding

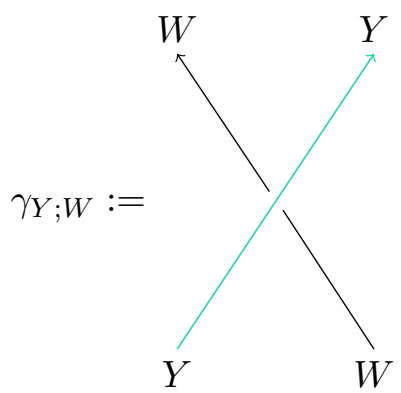

The definition of string-net spaces can be modified so that one assign to each boundary circle an object in the Drinfeld center $Z(\mathcal{C})$. We now give a working description of the extended stringnet spaces that are relevant to our construction of CFT correlators and refer to [17, Sections 6 and 7] for details. 
Remark 2.6. Let $S^{1}$ be an oriented circle. One can define a $\mathbb{K}$-linear category $\hat{\mathcal{C}}\left(S^{1}\right)$ whose objects are oriented circles with finite numbers of points labeled by objects of $\mathcal{C}$ and whose morphism space between two such circles are the string-net space on a cylinder with boundary value induced by the inclusion of the two circles as the boundary of the cylinder. The composition of morphisms are induced by the stacking of cylinders and the concatenation of string-nets, see [17, Definition 6.1] for details. For all $Y \in Z(\mathcal{C})$, the following string-net on a cylinder, considered as a morphism in $\hat{\mathcal{C}}\left(S^{1}\right)$, is a projector

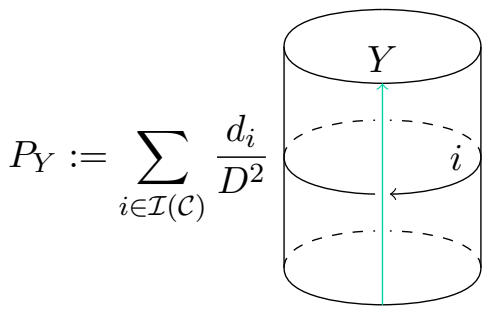

This can be seen by the following calculation in the string-net space, using the completeness relation in Proposition 2.2 and the naturality of the half-braiding

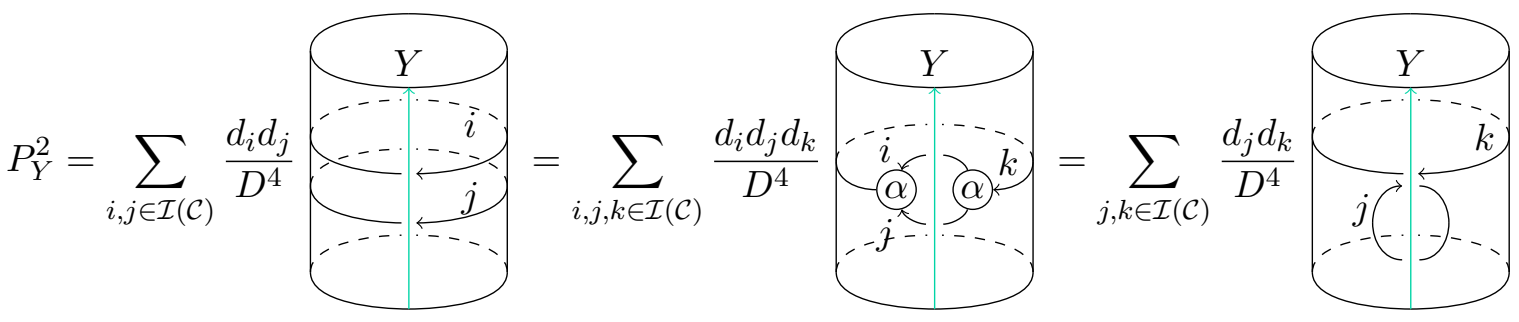

$$
\begin{aligned}
& =\sum_{k \in \mathcal{I}(\mathcal{C})} \frac{d_{k}}{D^{2}}
\end{aligned}
$$

We denote by an unoriented, unlabeled purple line the following morphism that is sometimes called the canonical color, the Kirby color, or the surgery color

$$
:=\sum_{i \in \mathcal{I}(\mathcal{C})} \frac{d_{i}}{D^{2}} \prod_{i} \in \operatorname{End}_{\mathcal{C}}\left(\bigoplus_{i \in \mathcal{I}(\mathcal{C})} X_{i}\right) .
$$

Therefore, the projector $P_{Y}$ can be also expressed as

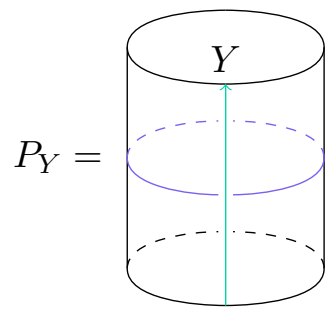

We are interested in the case where $\Sigma \cong \Sigma_{n}^{g}$, here $\Sigma_{n}^{g}$ means a compact oriented surface of genus $g$ with $n$ boundary components. Denote by $\left(\Sigma_{n}^{g}, Y_{1}, \ldots, Y_{n}\right)$ a $Z(\mathcal{C})$-marked surface, i.e., $\Sigma_{n}^{g}$ together with 
- a numbering of $\pi_{0}(\partial \Sigma)$ with $1, \ldots, n$,

- a choice of a point in each connected component of $\partial \Sigma$,

- a choice of $n$ objects $Y_{1}, \ldots, Y_{n} \in Z(\mathcal{C})$.

We denote the extended string-net space for the $Z(\mathcal{C})$-marked surface $\Sigma_{n}^{g}$ with this boundary value by $Z_{\mathrm{SN}, \mathcal{C}}\left(\Sigma_{n}^{g}, Y_{1}, \ldots, Y_{n}\right)$. This is defined to be a subspace of the (unextended) string-net spaces of $\Sigma_{n}^{g}$ with boundary value given by the underlying objects of $Y_{1}, \ldots, Y_{n}$ in $\mathcal{C}$, which is spanned by all the string-nets with the additional projectors introduced in Remark 2.6 placed near the corresponding boundary circles. For instance, a generic vector in $Z_{\mathrm{SN}, \mathcal{C}}\left(\Sigma_{3}^{1}, Y_{1}, Y_{2}, Y_{3}\right)$ can be defined by a linear combination of equivalence classes of colored graphs on $\Sigma_{3}^{1}$ such as

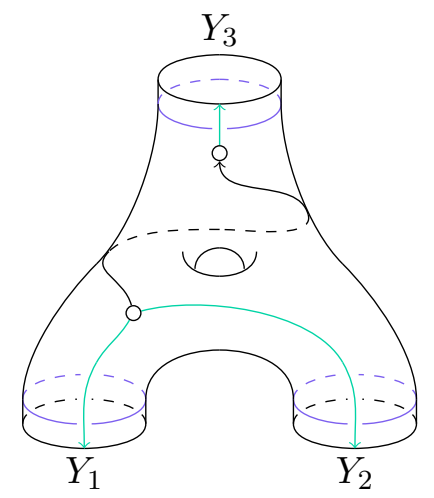

Figure 3. A generic string-net in $Z_{\mathrm{SN}, \mathcal{C}}\left(\Sigma_{3}^{1}, Y_{1}, Y_{2}, Y_{3}\right)$.

There is a canonical isomorphism

$$
Z_{\mathrm{SN}, \mathcal{C}}\left(\Sigma_{n}^{g}, Y_{1}, \ldots, Y_{n}\right) \cong Z_{\mathrm{TV}, \mathcal{C}}\left(\Sigma_{n}^{g}, Y_{1}, \ldots, Y_{n}\right),
$$

where $Z_{\mathrm{TV}, \mathcal{C}}\left(\Sigma_{n}^{g}, Y_{1}, \ldots, Y_{n}\right)$ is the state space for $\left(\Sigma_{n}^{g}, Y_{1}, \ldots, Y_{n}\right)$ in the extended Turaev-ViroBarrett-Westbury topological field theory [17]. Hence:

Proposition 2.7. There are isomorphisms

$$
Z_{\mathrm{SN}, \mathcal{C}}\left(\Sigma_{n}^{g}, Y_{1}, \ldots, Y_{n}\right) \cong Z_{\mathrm{TV}, \mathcal{C}}\left(\Sigma_{n}^{g}, Y_{1}, \ldots, Y_{n}\right) \cong \operatorname{Hom}_{Z(\mathcal{C})}\left(\mathbb{I}_{Z(\mathcal{C})}, Y_{1} \otimes \cdots \otimes Y_{n} \otimes L_{Z(\mathcal{C})}^{\otimes g}\right)
$$

that are functorial with respect to the morphisms in $Z(\mathcal{C})$, where $L_{Z(\mathcal{C})}:=\bigoplus_{i \in \mathcal{I}(Z(\mathcal{C}))} Z_{i}^{\vee} \otimes Z_{i}$.

\section{Bulk field correlators}

\subsection{Modular tensor categories}

The categorical ingredient of the string-net construction is a spherical fusion category $\mathcal{C}$, which is not necessarily braided. However, for the application to conformal field theories, we need a category with the structure of a ribbon fusion category over $\mathbb{C}$ with an additional nondegeneracy property:

Definition 3.1. A modular tensor category $\mathcal{C}$ is a ribbon fusion category over $\mathbb{C}$ with the braiding being nondegenerate in the sense that the matrix $\left(s_{i, j}\right)_{i, j \in \mathcal{I}(\mathcal{C})}$ is invertible, where $s_{i, j}:=$ $\operatorname{tr}\left(\beta_{j, i} \circ \beta_{i, j}\right)$.

It can be seen from the cyclic symmetry of the categorical trace that $s_{i, j}=s_{j, i}$. Moreover, one can show that (see, e.g., [2, Theorem 3.7.1])

$$
\sum_{k \in \mathcal{I}(\mathcal{C})} s_{i, k} s_{k, j}=D^{2} \delta_{i, \bar{j}} .
$$


For a spherical fusion category $\mathcal{C}$, the Drinfeld center $Z(\mathcal{C})$ is a modular tensor category.

We are going to use the following lemmas:

Lemma 3.2. For every $i \in \mathcal{I}(\mathcal{C})$, we have

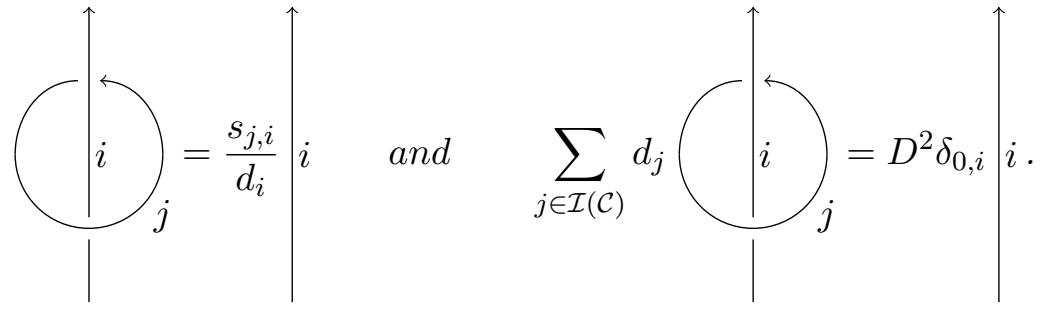

Lemma 3.3. For every $X \in \mathcal{C}$, we have

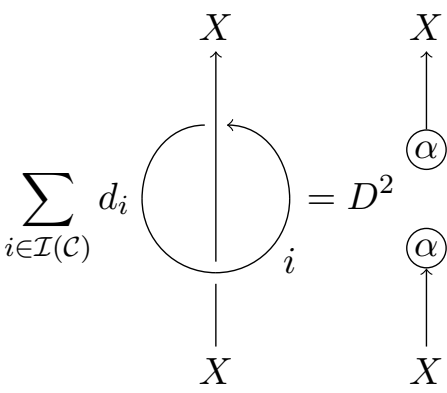

In particular, for every $i, j \in \mathcal{I}(\mathcal{C})$, we have

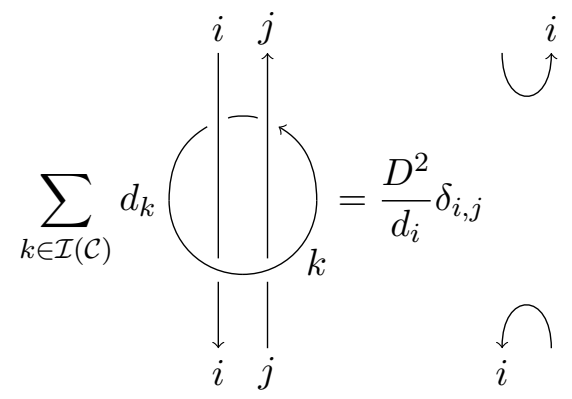

For a ribbon category $\mathcal{C}$, we denote by $\mathcal{C}^{\text {rev }}$ its reverse category, i.e., the same monoidal category with inverse braiding and twist. There is a canonical braided functor

$$
\begin{aligned}
\Xi: \mathcal{C}^{\mathrm{rev}} \otimes \mathcal{C} & \rightarrow Z(\mathcal{C}), \\
U \otimes V & \mapsto\left(U \otimes V, \gamma_{U \otimes V}^{\mathrm{uo}}\right),
\end{aligned}
$$

where

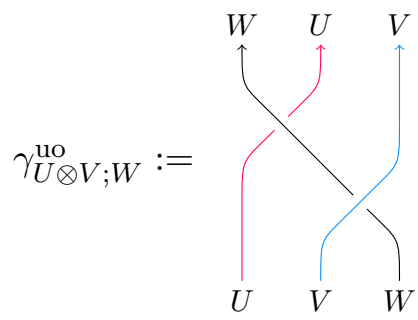

is the under-over half-braiding.

In fact, modularity can be formulated as follows, see, e.g., [24]:

Proposition 3.4. A ribbon fusion category $\mathcal{C}$ is a modular tensor category if and only if the canonical functor $\Xi$ is a braided equivalence. 


\subsection{Consistent systems of correlators}

We now give a summary of the concept of consistent systems of CFT bulk field correlators in the form used in [14].

An extended surface $\Sigma$ is an oriented surface with a partition of the boundary components into ingoing and outgoing parts, i.e., $\partial \Sigma=\partial_{\text {in }} \Sigma \sqcup \partial_{\text {out }} \Sigma$ and a marked point for each boundary component. We denote by $\Sigma_{p \mid q}^{g}$ an extended surface of genus $g$ with $p$ ingoing boundary components and $q$ outgoing boundary components.

Definition 3.5. The mapping class group $\operatorname{Map}(\Sigma)$ of an extended surface $\Sigma$ is the group of homotopy classes of orientation preserving homeomorphisms $\Sigma \rightarrow \Sigma$ that map $\partial_{\text {in }} \Sigma$ to itself (hence also $\partial_{\text {out }} \Sigma$ to itself) and map marked points to marked points.

Along with the action of the mapping class group on an extended surface, we also consider the sewing of the surface: A sewing $s_{\alpha, \beta}$ along $(\alpha, \beta) \in \pi_{0}\left(\partial_{\text {in }} \Sigma\right) \times \pi_{0}\left(\partial_{\text {out }} \Sigma\right)$ gives us a new extended surface $s_{\alpha, \beta}(\Sigma):=\cup_{\alpha, \beta} \Sigma$ by identifying the boundary component $\partial_{\alpha} \Sigma$ with $\partial_{\beta} \Sigma$ via an orientation preserving homeomorphism $f: \partial_{\alpha} \Sigma \rightarrow \partial_{\beta} \Sigma$ that maps the marked point on $\partial_{\alpha} \Sigma$ to the marked point on $\partial_{\beta} \Sigma$. The resulting surface is independent of $f$ up to homeomorphisms.

Definition 3.6. The category $\mathcal{S}$ urf is the symmetric monoidal category having extended surfaces $\Sigma$ as objects and the pairs $\left(\varphi, s_{\alpha, \beta}\right)$ as morphisms $\Sigma \rightarrow \cup_{\alpha, \beta} \Sigma$, where $\varphi \in \operatorname{Map}(\Sigma)$ is a mapping class and $s_{\alpha, \beta}$ a sewing. The monoidal product is given by the disjoint union.

In order to describe the composition of the morphisms in the category $\mathcal{S}$ urf, we need the relations among the pairs of mapping classes and sewing. Such relations are discussed in detail in $[16]$.

In a local two-dimensional conformal field theory, specific spaces of conformal blocks for bulk fields can be constructed as the morphism spaces in a braided monoidal category $\mathcal{D}$ involving a fixed object $F \in \mathcal{D}$. The object $F \in \mathcal{D}$ should be considered as the space of bulk fields. We say that the CFT has the monodromy data based on $\mathcal{D}$ and the bulk object $F$. The reader is invited to think of $\mathcal{D}$ as the representation category of both left moving and right moving chiral symmetries. The collection of all bulk fields transforms in a representation of this symmetry which also determines the monodromy data of the theory like braiding and fusing matrices. Hence we say that the CFT has the monodromy data based on $\mathcal{D}$ and the bulk object $F$ describing all bulk fields.

Since we are interested in correlators of bulk fields, we consider conformal blocks that are based on the modular tensor category $\mathcal{D}=\mathcal{C}^{\text {rev }} \otimes \mathcal{C}$ for a modular tensor category $\mathcal{C}$ (correlators of bulk fields are obtained by combining conformal blocks for left movers with those for right movers). Because of Proposition 3.4, we can replace $\mathcal{D}=\mathcal{C}^{\text {rev }} \otimes \mathcal{C}$ with the Drinfeld center $Z(\mathcal{C})$ and therefore apply the Turaev-Viro-Barrett-Westbury state-sum construction, or equivalently, the string-net model described in Section 2.

We therefore define the pinned block functor

$$
\mathrm{Bl}^{F}: \mathcal{S} \text { urf } \rightarrow \mathcal{V} \operatorname{ect}_{\mathbb{C}}
$$

by assigning to the extended surface $\Sigma_{p \mid q}^{g}$ the finite dimensional vector space

$$
\mathrm{Bl}^{F}\left(\Sigma_{p \mid q}^{g}\right):=Z_{\mathrm{SN}, \mathcal{C}}(\Sigma_{p+q}^{g}, \underbrace{F^{\vee}, \ldots, F^{\vee}}_{p}, \underbrace{F, \ldots, F}_{q}) \cong \operatorname{Hom}_{Z(\mathcal{C})}\left(\mathbb{I}_{Z(\mathcal{C})},\left(F^{\vee}\right)^{\otimes p} \otimes F^{\otimes q} \otimes L_{Z(\mathcal{C})}^{\otimes g}\right),
$$

and to a morphism $(\varphi, s)$ between extended surfaces the natural action of the mapping class $\varphi$ followed by the concatenation of the string-net induced by the sewing $s$. 
As an auxiliary datum, we also define the trivial block functor $\Delta_{\mathbb{C}}:$ Surf $\rightarrow \mathcal{V}_{e} t_{\mathbb{C}}$ by assigning to every extended surface the vector space $\mathbb{C}$ and to every morphism the identity id $\mathbb{C}$. A consistent system of bulk field correlators is then a monoidal natural transformation

$$
v^{F}: \Delta_{\mathbb{C}} \rightarrow \mathrm{Bl}^{F}
$$

such that $v^{F}\left(\Sigma_{1 \mid 1}^{0}\right):=\left(v^{F}\right)_{\Sigma_{1 \mid 1}^{0}}(1) \in \operatorname{Bl}^{F}\left(\Sigma_{1 \mid 1}^{0}\right) \cong \operatorname{End}_{Z(\mathcal{C})}(F)$ is invertible.

Unpacking the rather compact definition above, we see that the so defined consistent system of bulk field correlators amounts to a choice of a vector

$$
v^{F}\left(\Sigma_{p \mid q}^{g}\right):=\left(v^{F}\right)_{\Sigma_{p \mid q}^{g}}(1) \in \operatorname{Bl}^{F}\left(\Sigma_{p \mid q}^{g}\right)
$$

for each extended surface $\Sigma_{p \mid q}^{g}$ that is invariant under the action of the mapping class group $\operatorname{Map}\left(\Sigma_{p \mid q}^{g}\right)$, such that the linear map induced by a sewing maps the chosen vector to the chosen vector for the sewn surface.

It is shown [14, Theorem 4.10] that for a (not necessarily semisimple) modular finite category $\mathcal{D}$, the consistent systems of bulk field correlators with monodromy data based on $\mathcal{D}$ and with bulk object $F \in \mathcal{D}$ are in bijection with structures of a modular Frobenius algebra [14, Definition 4.9] on $F$.

\subsection{Fundamental correlators via string-nets}

Let $F \in Z(\mathcal{C})$ be a commutative symmetric Frobenius algebra. We define the following stringnets on the set of surfaces that generates all extended surfaces by sewing
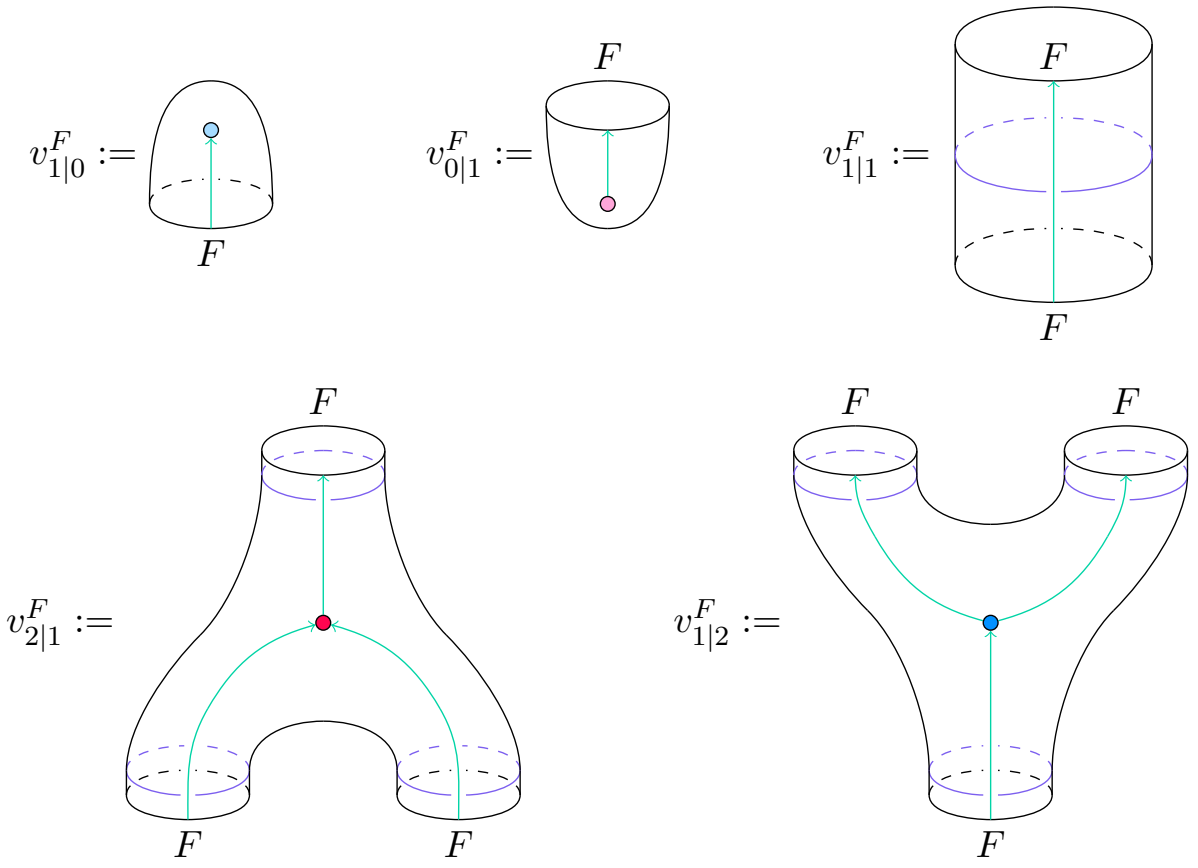

Here the vertices are given by the coproduct of the Frobenius algebra $F$. A priori, our prescription depends on the isotopy classes of the embeddings of the string diagrams into the surfaces. However, by using the following cloaking relation, we can show that the string-nets above are in fact well defined:

Lemma 3.7. Let $\Sigma$ be a compact oriented surface, $\mathbf{V}$ a boundary value, and $X, Y \in Z(\mathcal{C})$. We have the following equation in the string-net space $Z_{\mathrm{SN}, \mathcal{C}}(\Sigma, \mathbf{V})$ 


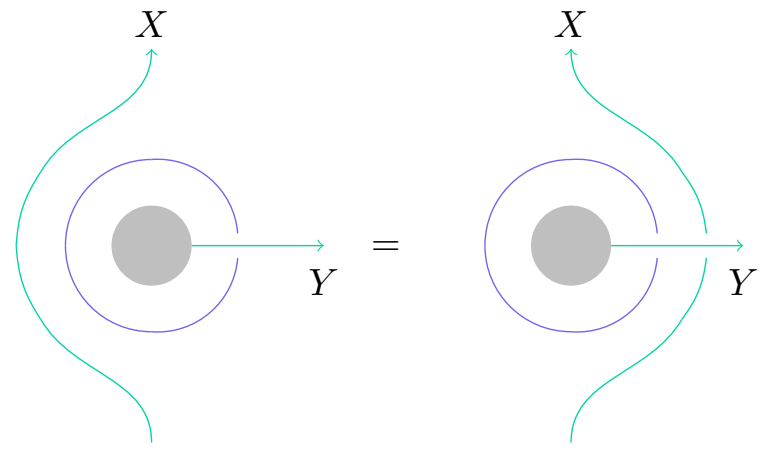

here it is understood that the string-nets agree outside the depicted region, and the shaded area may include boundary components.

Proof. By using Proposition 2.2 and the naturality of the half-braiding, we can see that both sides are equal to

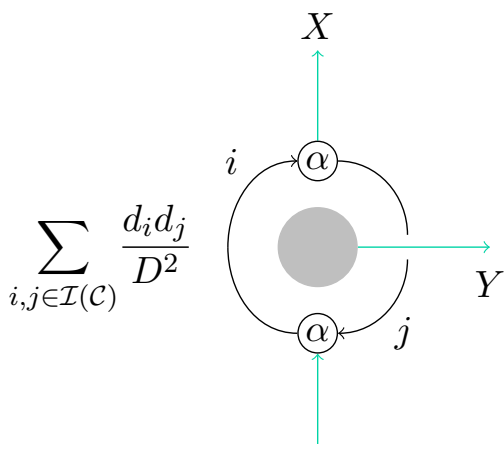

Lemma 3.8. For a commutative symmetric Frobenius algebra $F \in Z(\mathcal{C}), v_{1 \mid 0}^{F}, v_{0 \mid 1}^{F}, v_{1 \mid 1}^{F}, v_{2 \mid 1}^{F}$, and $v_{1 \mid 2}^{F}$ are invariant under the mapping class groups.

Proof. The cases of $v_{1 \mid 0}^{F}, v_{0 \mid 1}^{F}$ are trivial, since the mapping class group of a disc is trivial.

The mapping class group of a cylinder is generated by a Dehn twist. By doing a Dehn twist $T$ along the projector on the cylinder, we have:

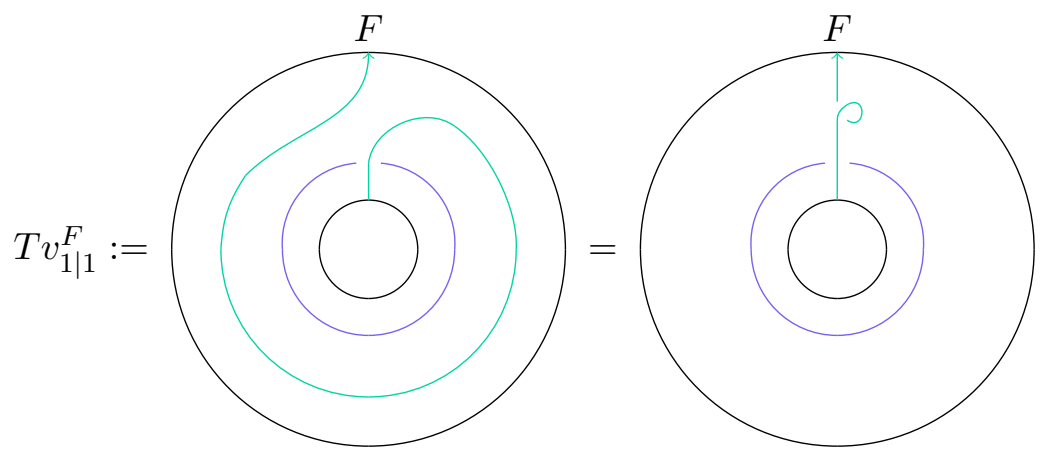

Here we have used the cloaking relation in Proposition 3.7. Since being commutative and symmetric implies that $F$ has trivial twist [8, Proposition 2.25], we have $T v_{1 \mid 1}^{F}=v_{1 \mid 1}^{F}$.

Now consider the so-called $B$ move on a pair of pants with two ingoing boundary components, we have 


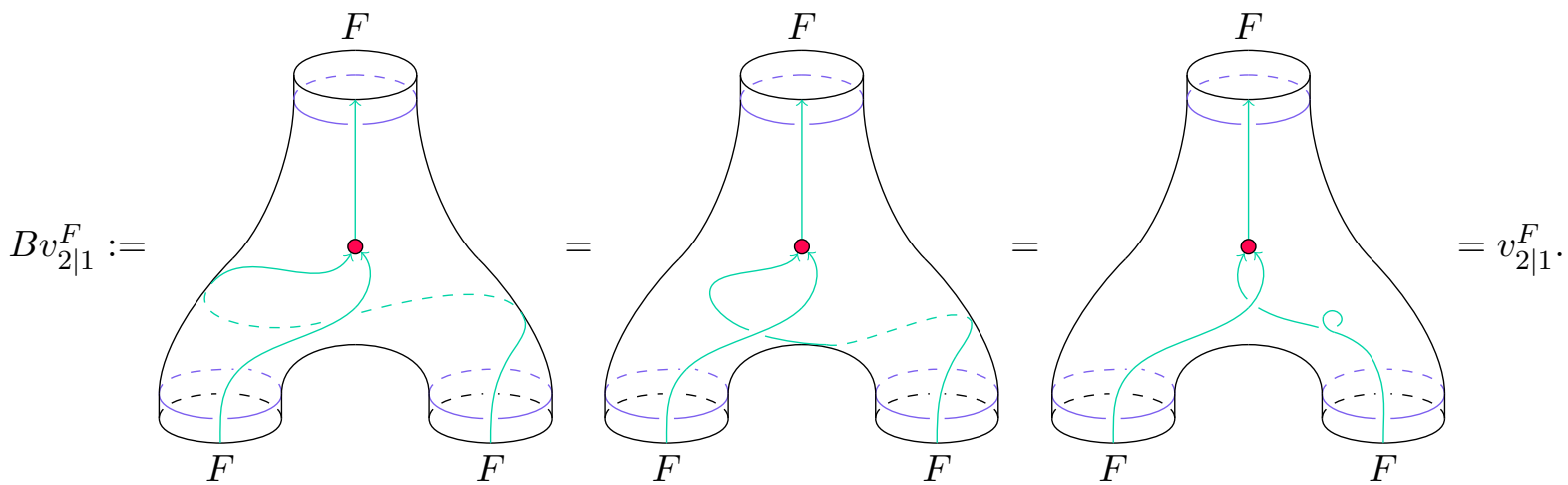

Here we have used the cloaking relation twice on the line ending on the lower right circle and then the commutativity and symmetry of $F$. Since the $B$ move and the Dehn twists along the boundary circles generate the mapping class group, we have shown the invariance. The invariance of $v_{1 \mid 2}^{F}$ can be shown in a similar way by using cocommutativity of $F$.

By setting $v^{F}\left(\Sigma_{1 \mid 0}^{0}\right)=v_{1 \mid 0}^{F}, v^{F}\left(\Sigma_{0 \mid 1}^{0}\right)=v_{0 \mid 1}^{F}, v^{F}\left(\Sigma_{1 \mid 1}^{0}\right)=v_{1 \mid 1}^{F}, v^{F}\left(\Sigma_{2 \mid 1}^{0}\right)=v_{2 \mid 1}^{F}, v^{F}\left(\Sigma_{1 \mid 2}^{0}\right)=v_{1 \mid 2}^{F}$, we can extend our prescription to a consistent system of correlators via sewing, provided that the string-net we get on the torus with one boundary component is invariant under the action of the mapping class group. The argument is essentially the same as the one used in [14], i.e., via considering the Lego-Teichmüller game [1]. Notice that the consistency regarding surfaces of genus zero follows purely from the fact that $F$ is a commutative symmetric Frobenius algebra in $Z(\mathcal{C})$ and cloaking. For instance, since we can move the projectors around by using the cloaking relation, the Frobenius property implies

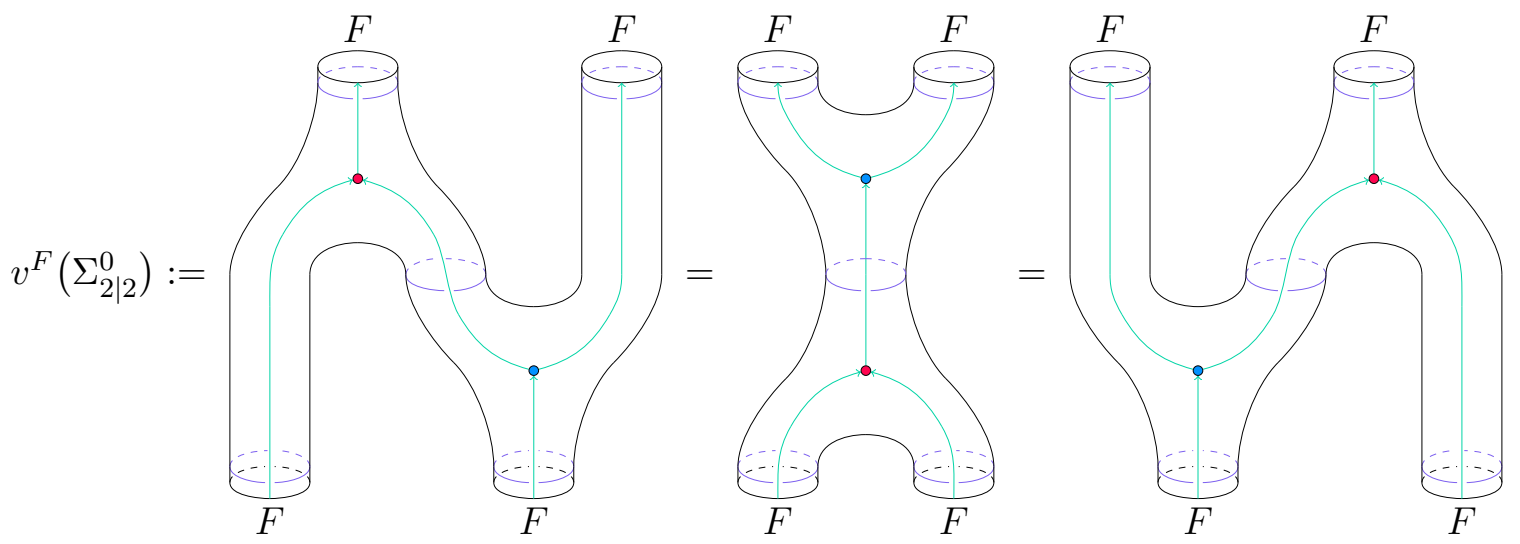

It was shown in [25, Lemma 6.6] that the condition of $S$-invariance of the string-net on the torus with one boundary circle corresponds to the $S$-invariance condition in [21, Lemma 3.2], which is equivalent to the modularity condition of the Frobenius algebra given in [14, Definition 4.9] in the semi-simple cases. The surprising result [21, Theorem 3.4] states that a haploid commutative symmetric Frobenius algebra $F \in Z(\mathcal{C})$ is modular if and only if $\operatorname{dim}(F)=D^{2}$.

\section{The Cardy case}

\subsection{The bulk algebra for the Cardy case}

So far, we have been working with a general commutative symmetric Frobenius algebra. We now consider a specific Frobenius algebra, which is the algebra of the bulk fields in the Cardy case.

We equip the object

$$
L:=\bigoplus_{i \in \mathcal{I}(\mathcal{C})} X_{i}^{\vee} \otimes X_{i}
$$




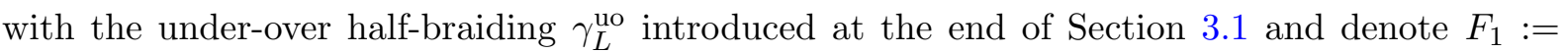
$\left(L, \gamma_{L}^{\mathrm{uo}}\right) \in Z(\mathcal{C})$ in the following.

We next recall that it has a natural Frobenius algebra structure in $Z(\mathcal{C})$, see a review $[9$, Section 2.2] and references therein.

Proposition 4.1. $\left(F_{1}, \mu_{F_{1}}, \eta_{F_{1}}, \Delta_{F_{1}}, \varepsilon_{F_{1}}\right)$ is a commutative, symmetric Frobenius algebra in $Z(\mathcal{C})$, where

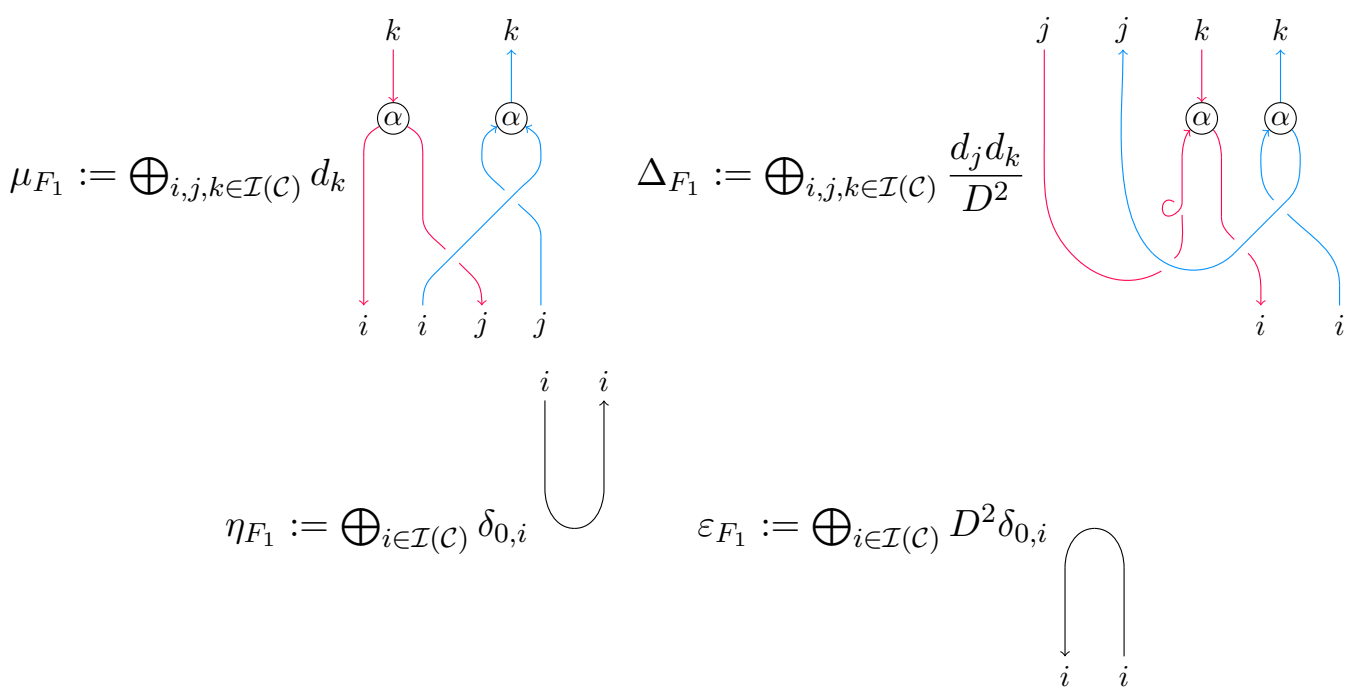

In order to show that the prescription given in the Section 3.3 for the Frobenius algebra $F_{1}$ extends to a consistent system of correlators, we need to show that the string-net we get on the torus with one boundary circle is invariant under the mapping class group action. This is guaranteed by its dimension according to [21, Theorem 3.4]. However, the consistency for the Cardy case can be seen in a much more straight forward and geometric manner, and a closed form of the correlators can be derived: it turns out that the string-nets we get, in their most simplified forms, are as empty as possible.

\subsection{Consistency made explicit}

The coend $L=\bigoplus_{i \in \mathcal{I}(\mathcal{C})} X_{i}^{\vee} \otimes X_{i}$ can be also equipped with a different half-braiding that can be understood from the central monad

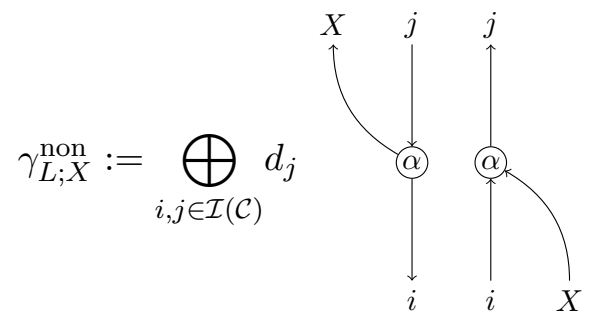

We call it the non-crossing half-braiding for the obvious reason.

We denote $\widetilde{F}:=\left(L, \gamma_{L}^{\text {non }}\right) \in Z(\mathcal{C})$. There is also a naturally defined Frobenius algebra structure on this object, with the multiplication and co-multiplication given by
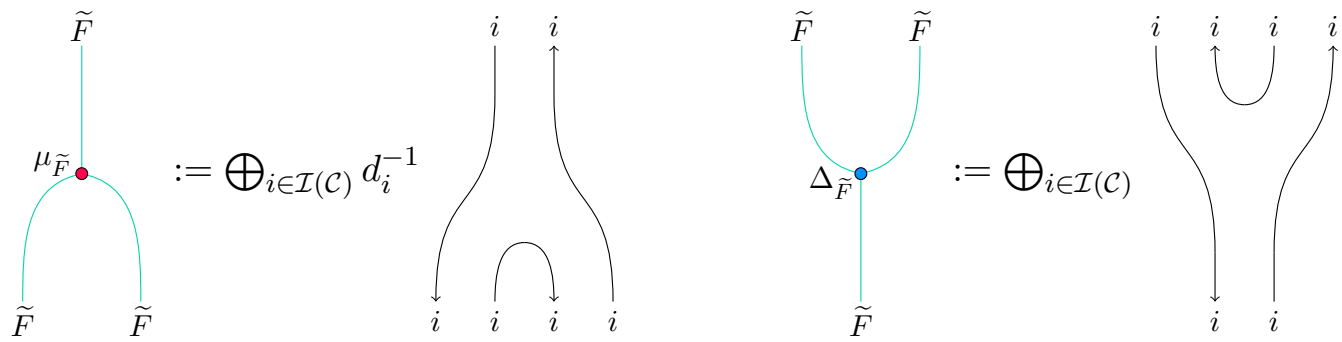
It is easy to show that this is a special symmetric Frobenius algebra.

Proposition 4.2. For a modular tensor category $\mathcal{C}$, the morphism

$$
S_{L}:=\bigoplus_{i, j \in \mathcal{I}(\mathcal{C})} d_{j} \underbrace{j}_{\substack{i \\ i}} \in \operatorname{End}_{\mathcal{C}}(L)
$$

is an isomorphism of Frobenius algebras in $Z(\mathcal{C})$ from the Cardy bulk algebra $\left(F_{1}, \mu_{F_{1}}, \eta_{F_{1}}\right.$, $\left.\Delta_{F_{1}}, \varepsilon_{F_{1}}\right)$ in Proposition 4.1 to the Frobenius algebra $\left(\widetilde{F}, \mu_{\widetilde{F}}, \eta_{\widetilde{F}}, \Delta_{\widetilde{F}}, \varepsilon_{\widetilde{F}}\right)$ defined above, with the inverse given by

$$
S_{L}^{-1}:=\bigoplus_{i, j \in \mathcal{I}(\mathcal{C})} \frac{d_{j}}{D^{2}} \overbrace{i}^{j}
$$

Proof. A more general form of the fact that the given morphisms are isomorphisms of the Frobenius algebras (regarded as Frobenius algebras in $\mathcal{C}$ ) was proven in [20, Proposition 4.3]. We present here a simple proof of the special case we need. Note that this can be generalized to non-semisimple settings.

Using Proposition 2.2, it is not hard to see that

$$
S_{L} \in \operatorname{Hom}_{Z(\mathcal{C})}\left(F_{1}, \widetilde{F}\right) \quad \text { and } \quad S_{L}^{-1} \in \operatorname{Hom}_{Z(\mathcal{C})}\left(\widetilde{F}, F_{1}\right)
$$

For instance

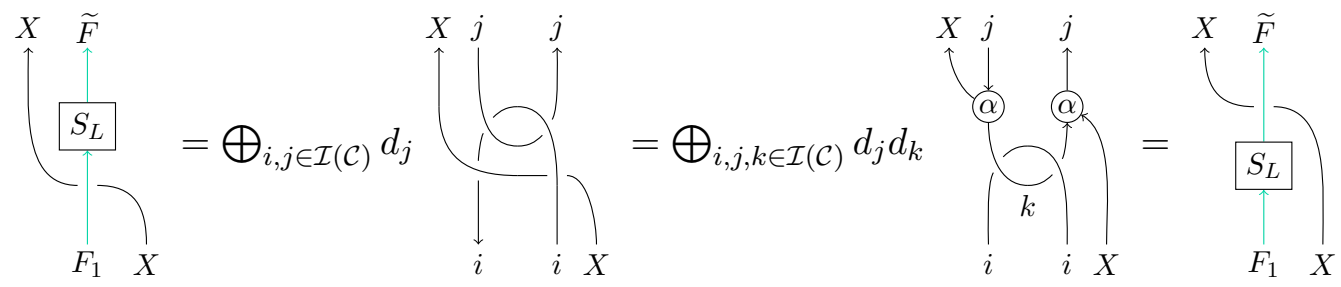

The fact that $S_{L}$ and $S_{L}^{-1}$ are inverse to each other is equivalent to Lemma 3.3.

To show that $S_{L}$ is an isomorphism of algebras, we notice
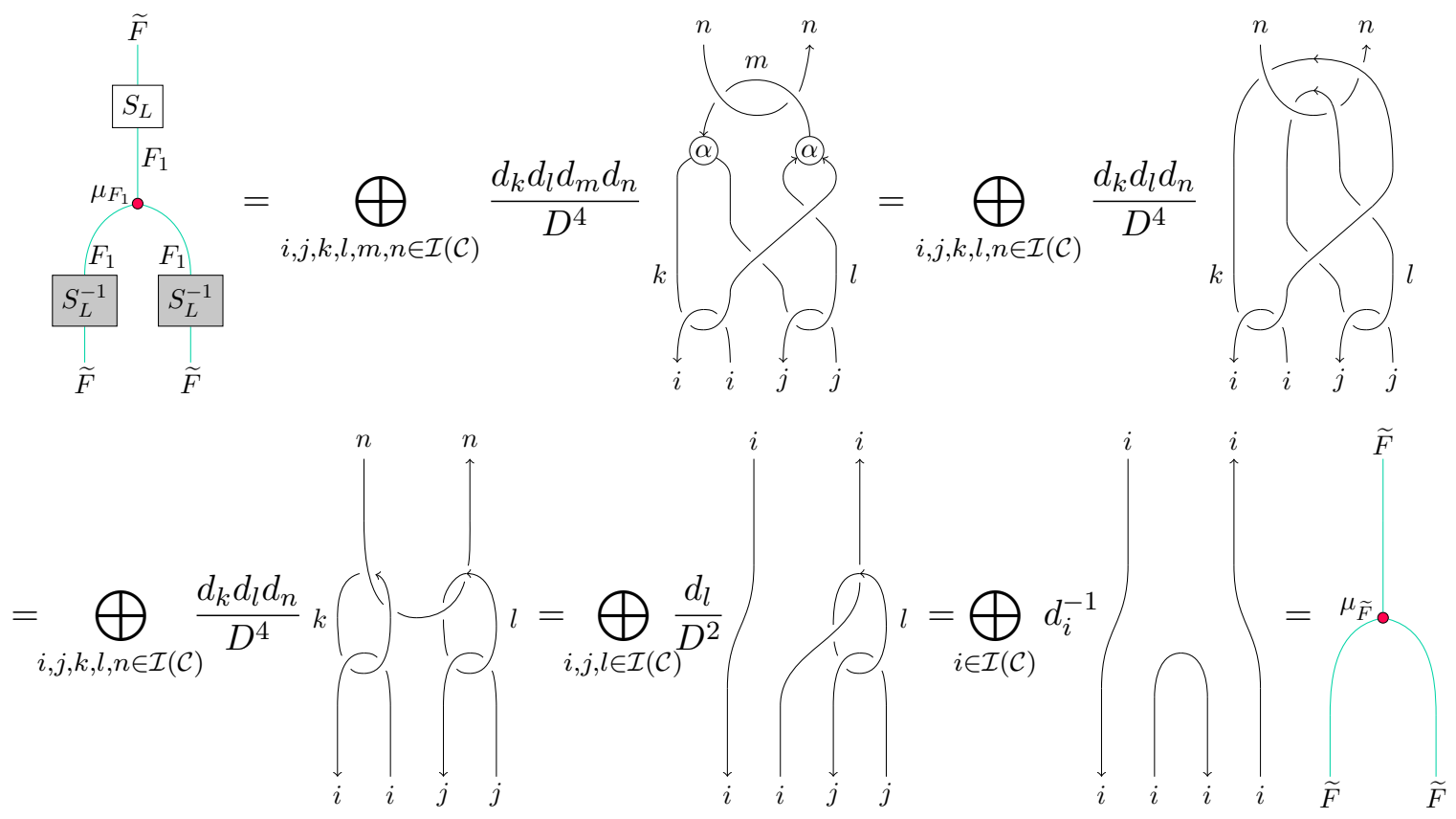
Hence $S_{L} \circ \mu_{F}=\mu_{\widetilde{F}} \circ\left(S_{L} \otimes S_{L}\right)$. Similarly, one shows that $S_{L}$ is also an isomorphism of coalgebras
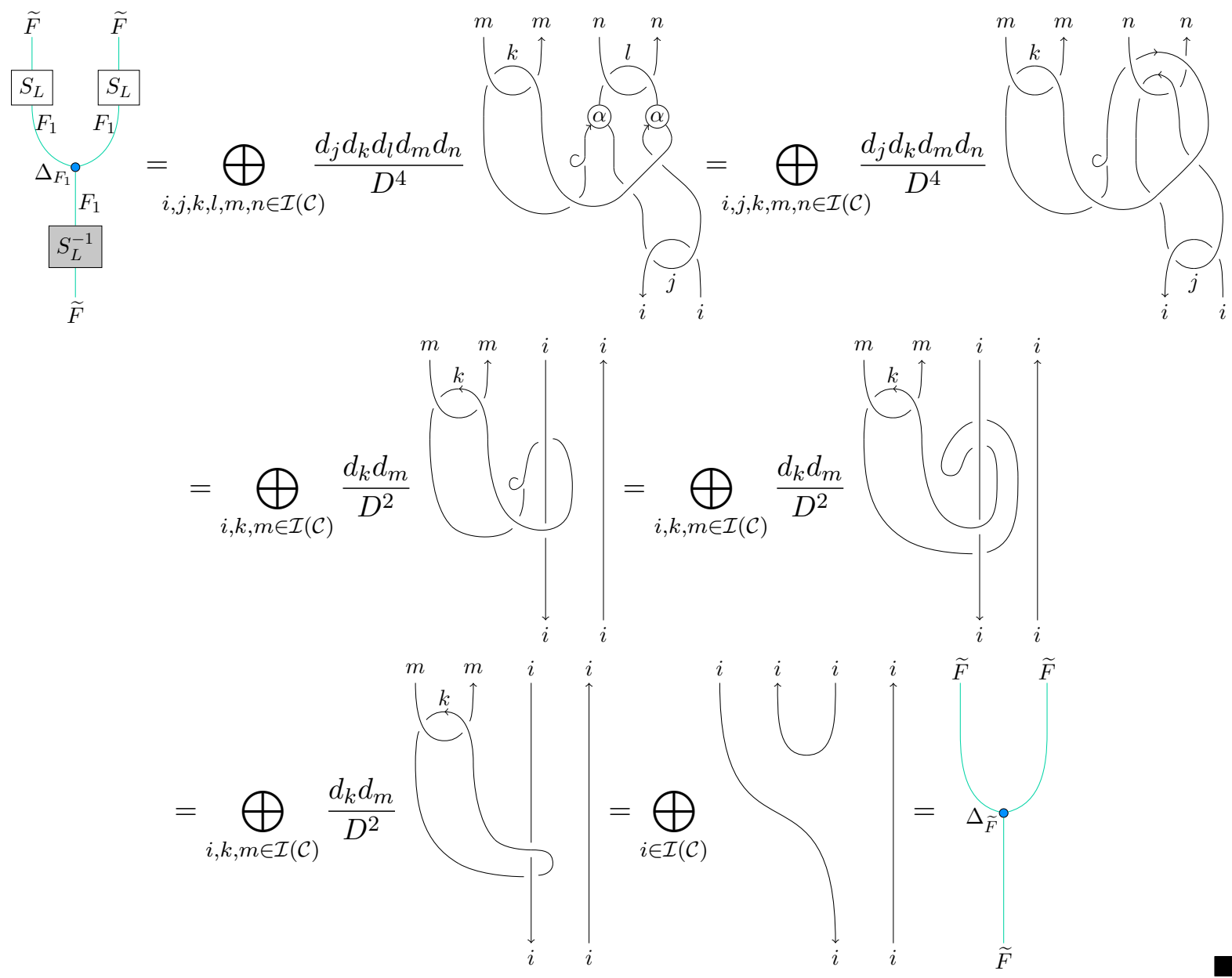

Corollary 4.3. For a modular tensor category $\mathcal{C},\left(\widetilde{F}, \mu_{\widetilde{F}}, \eta_{\widetilde{F}}, \Delta_{\widetilde{F}}, \varepsilon_{\widetilde{F}}\right)$ is a commutative, symmetric Frobenius algebra in $Z(\mathcal{C})$. In particular, $\widetilde{F}$ has trivial twist.

Remark 4.4. In fact, Corollary 4.3 holds true for any spherical fusion category $\mathcal{C}$.

It is a general fact that isomorphisms between bulk algebras induce isomorphisms of the spaces of conformal blocks. In the case at hand, this is implemented by composing the string-nets with the morphism $S_{L}$ near the outgoing boundary and precomposing the string-net with $S_{L}^{-1}$ near the ingoing boundary. For instance, applying to the invariants on pairs of pants, we get

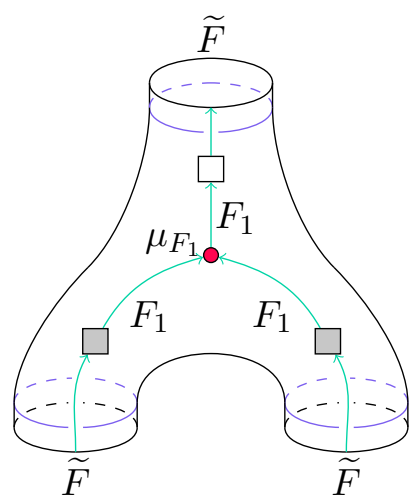

and

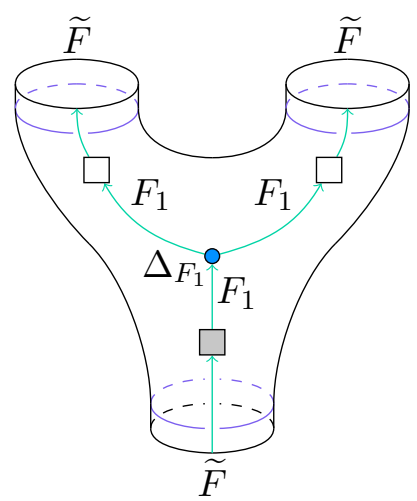


Here the white boxes stand for $S_{L}$ and the gray ones stand for $S_{L}^{-1}$. Since both are morphisms in $Z(\mathcal{C})$, it makes no difference which side of the projectors we put the boxes on, as long as we use the correct half-braidings.

If we take $\widetilde{F}$ as our bulk object, we get another set of conformal blocks

$$
\mathrm{Bl}^{\widetilde{F}}: \mathcal{S} \text { urf } \rightarrow \mathcal{V} \operatorname{ect}_{\mathbb{C}}
$$

as well as a new set of correlators

$$
v^{\widetilde{F}}: \Delta_{\mathbb{C}} \rightarrow \mathrm{Bl}^{\widetilde{F}} .
$$

In fact, the induced isomorphisms of string-net spaces give rise to a natural isomorphisms of conformal blocks

$$
\mathrm{Bl}^{S_{L}}: \mathrm{Bl}^{F_{1}} \rightarrow \mathrm{Bl}^{\widetilde{F}}
$$

since the isomorphisms intertwine the action of mapping class groups and sewing.

Moreover, due to the fact that

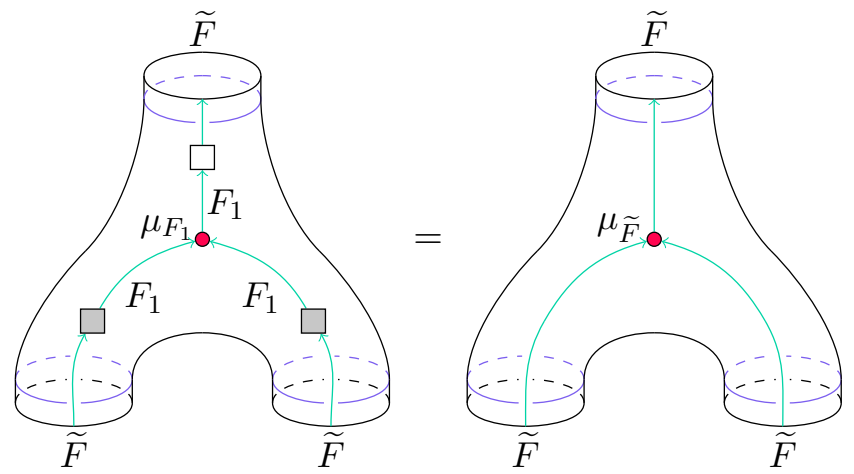

and

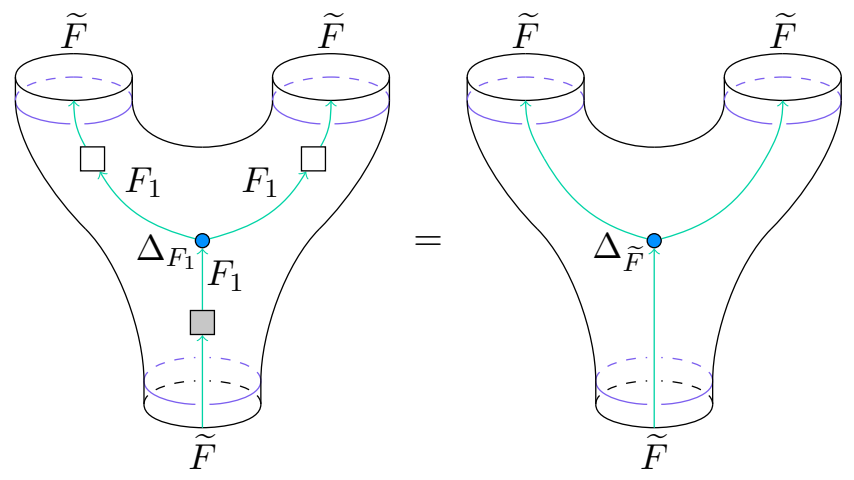

we get a commutative diagram of natural transformations

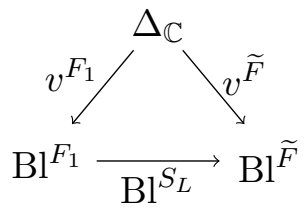

Intuitively, the two isomorphic Frobenius algebras produce equivalent sets of correlators. The natural isomorphism $\mathrm{Bl}^{S_{L}}$ gives the precise way to relate them.

It turns out that the correlators given by the Frobenius algebra $\left(\widetilde{F}, \mu_{\widetilde{F}}, \eta_{\widetilde{F}}, \Delta_{\widetilde{F}}, \varepsilon_{\widetilde{F}}\right)$ are particularly easy to compute:

Theorem 4.5. Let $\Sigma_{p \mid q}^{g}$ be a surface of genus $g$ with $p$ ingoing and $q$ outgoing boundaries, where $p, q, g \in \mathbb{Z}_{\geq 0}$. 
1. The correlator for $\Sigma_{p \mid q}^{g}$ with bulk field $\widetilde{F}$ is given by the following string net:

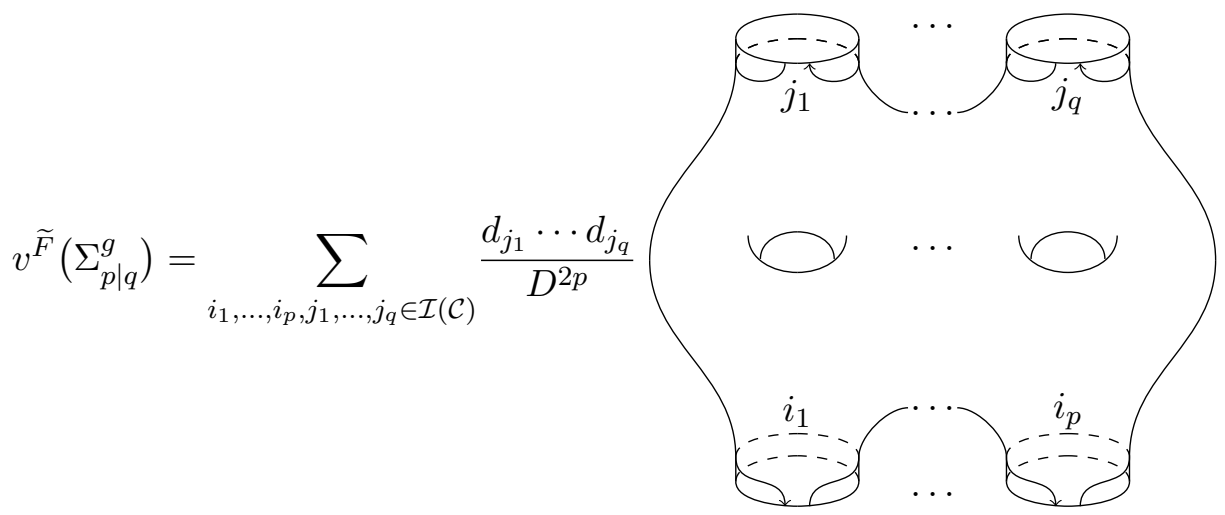

2. Consequently, the correlator for $\Sigma_{p \mid q}^{g}$ with bulk field $F_{1}$ is given by the following string net:

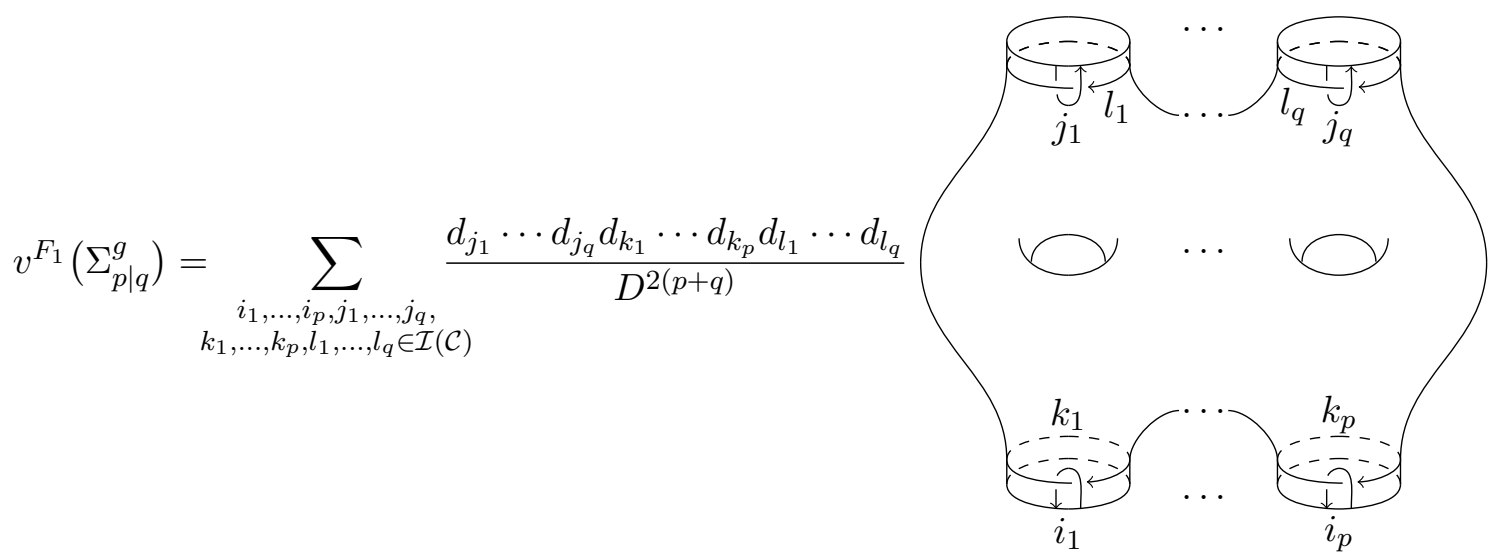

Proof. We only have to check the cases in which $g=0$ and $p+q \leq 3$. For $\Sigma_{2 \mid 1}^{0}$, we find

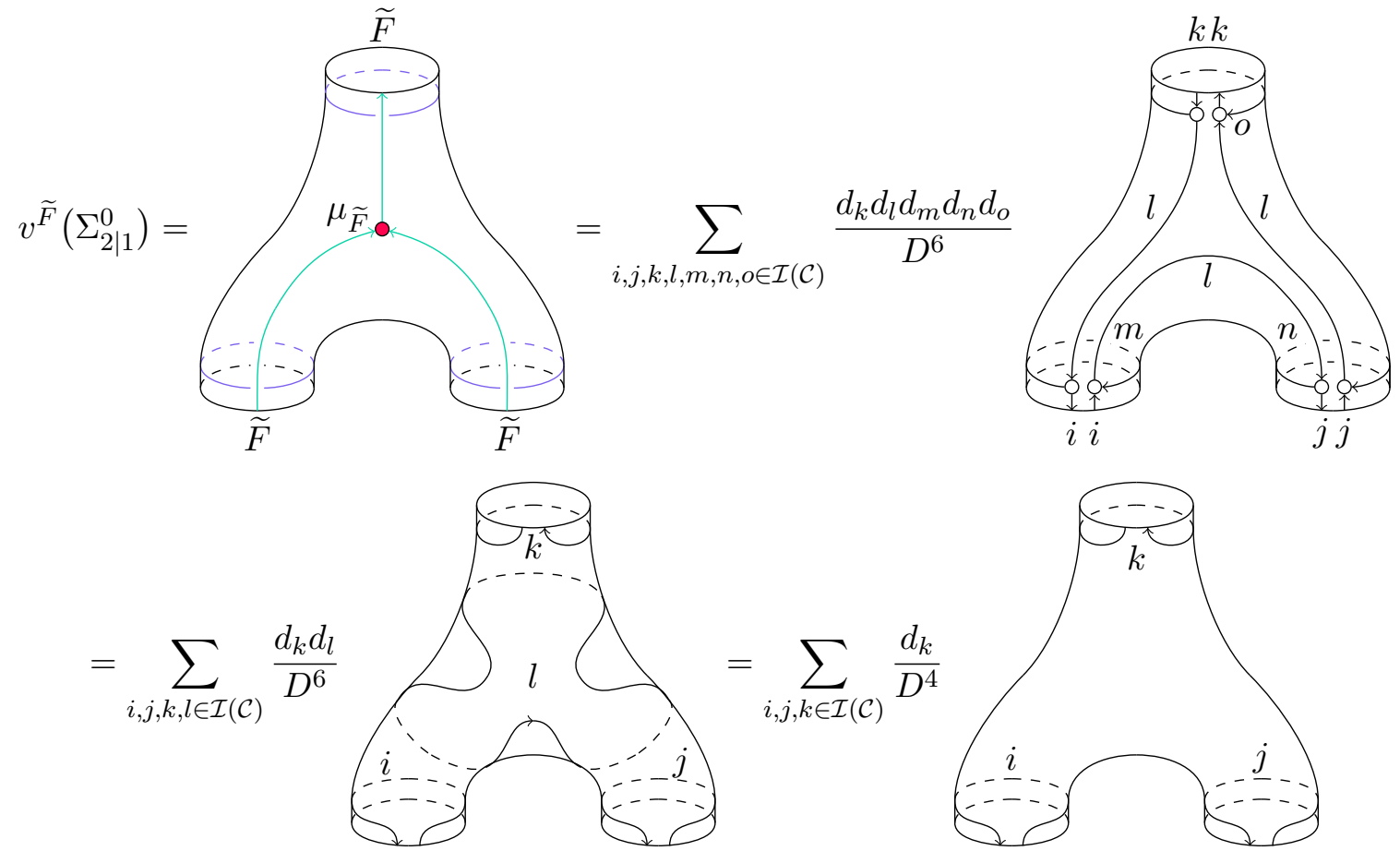


Similarly, we have

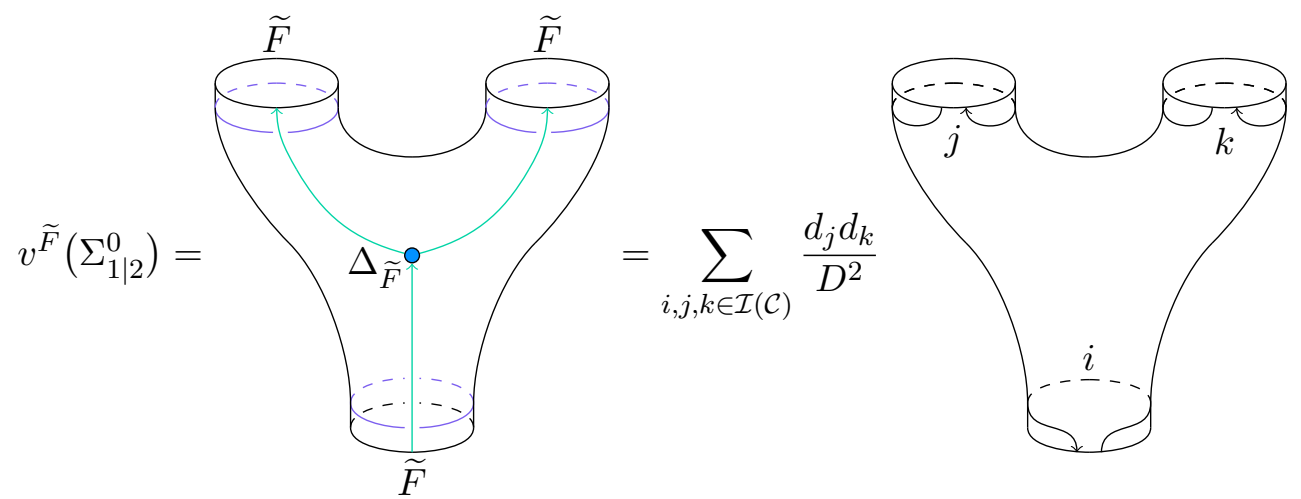

The arguments concerning the unit and counit are even more straight forward. Notice that, whenever we sew together a pair of boundaries, we get a contractible circle that cancels out a factor of $D^{2}$.

By applying the inverse of the natural isomorphism $\mathrm{Bl}^{S_{L}}$, we get the second part of the statement.

Remark 4.6. It can be seen from the proof above, even though the definition of the Frobenius algebra $F_{1}$ requires $\mathcal{C}$ to be braided and the construction of the isomorphisms in Proposition 4.2 requires $\mathcal{C}$ to be modular, the Frobenius algebra $\widetilde{F} \in Z(\mathcal{C})$ gives rise to a consistent system of bulk field correlators for any spherical fusion category $\mathcal{C}$.

Theorem 4.5 allows us to compute in particular the zero-point correlator on a torus. Consider the following set of vectors $\left\{G_{i, j}\right\}_{i, j \in \mathcal{I}(\mathcal{C})}$ in the string-net space of a torus, where

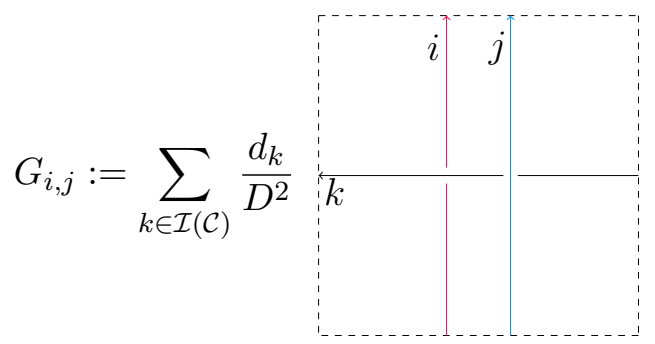

here the opposite sides of the square are identified so the resulting surface is a torus. For a modular tensor category $\mathcal{C}$, every simple object in $Z(\mathcal{C}) \simeq \mathcal{C}^{\text {rev }} \otimes \mathcal{C}$ is isomorphic to $Z_{(i, j)}:=$ $\left(X_{i} \otimes X_{j}, \gamma_{(i, j)}\right)$ for some $i, j \in \mathcal{I}(\mathcal{C})$ and the half braiding $\gamma_{(i, j)}$ given by the under-over halfbraiding. It can be seen from the following representation of the string-net space associated to the torus

$$
Z_{\mathrm{SN}, \mathcal{C}}\left(\Sigma_{0}^{1}\right) \cong \bigoplus_{k \in \mathcal{I}(Z(\mathcal{C}))} Z_{\mathrm{SN}, \mathcal{C}}\left(\Sigma_{2}^{0}, Z_{k}^{\vee}, Z_{k}\right) \cong \bigoplus_{i, j \in \mathcal{I}(\mathcal{C})} Z_{\mathrm{SN}, \mathcal{C}}\left(\Sigma_{2}^{0}, Z_{(i, j)}^{\vee}, Z_{(i, j)}\right)
$$

that follows from factorization that $\left\{G_{i, j}\right\}_{i, j \in \mathcal{I}(\mathcal{C})}$, up to the action of the mapping class group $\operatorname{Map}\left(\Sigma_{0 \mid 0}^{1}\right) \cong \operatorname{SL}(2, \mathbb{Z})$, is a basis for the vector space $Z_{\mathrm{SN}, \mathcal{C}}\left(\Sigma_{0}^{1}\right)$. As a result of Theorem 4.5 , the torus partition function $v^{F_{1}}\left(\Sigma_{0 \mid 0}^{1}\right)$ is the empty string-net, which is obviously invariant under the mapping class group action. When written in the following form

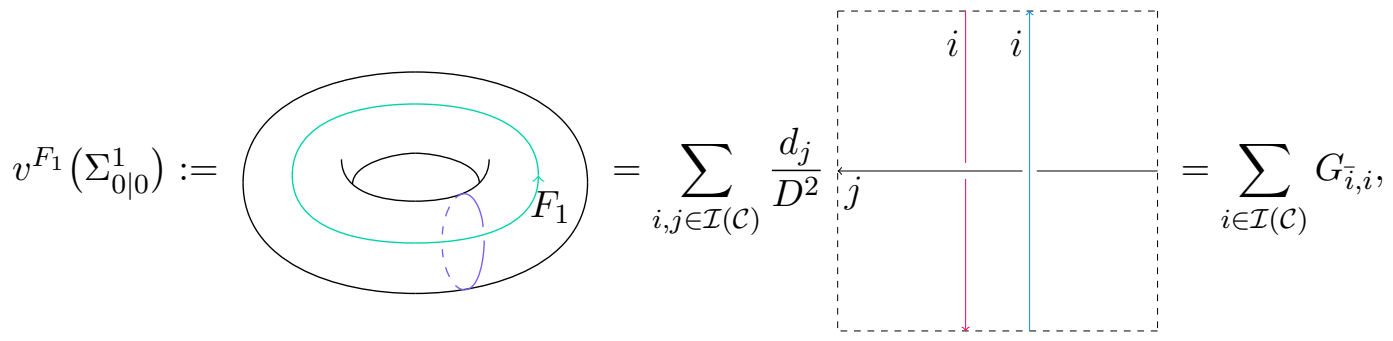


the correlator is expressed as a linear combination of the basis vectors $\left\{G_{i, j}\right\}_{i, j \in \mathcal{I}(\mathcal{C})}$ with the coefficients $\left(\delta_{i, j}\right)_{i, j \in \mathcal{I}(\mathcal{C})}$, which are the entries of the charge conjugation matrix.

\section{Acknowledgements}

We thank Alain Bruguières, Jürgen Fuchs, Eilind Karlsson and Vincentas Mulevičius for helpful discussions. The authors are partially supported by the RTG 1670 "Mathematics inspired by String theory and Quantum Field Theory" and by the Deutsche Forschungsgemeinschaft (DFG, German Research Foundation) under Germany's Excellence Strategy - EXC 2121 "Quantum Universe"-QT.2.

\section{References}

[1] Bakalov B., Kirillov Jr. A., On the Lego-Teichmüller game, Transform. Groups 5 (2000), 207-244, arXiv:math.GT/9809057.

[2] Bakalov B., Kirillov Jr. A., Lectures on tensor categories and modular functors, University Lecture Series, Vol. 21, Amer. Math. Soc., Providence, RI, 2001.

[3] Balsam B., Turaev-Viro theory as an extended TQFT II, arXiv:1010.1222.

[4] Balsam B., Turaev-Viro theory as an extended TQFT III, arXiv:1012.0560.

[5] Etingof P., Nikshych D., Ostrik V., On fusion categories, Ann. of Math. 162 (2005), 581-642, arXiv:math.QA/0203060.

[6] Felder G., Fröhlich J., Fuchs J., Schweigert C., Correlation functions and boundary conditions in rational conformal field theory and three-dimensional topology, Compositio Math. 131 (2002), 189-237, arXiv:hepth/9912239.

[7] Fjelstad J., Fuchs J., Runkel I., Schweigert C., TFT construction of RCFT correlators. V. Proof of modular invariance and factorisation, Theory Appl. Categ. 16 (2006), 16, 342-433, arXiv:hep-th/0503194.

[8] Fröhlich J., Fuchs J., Runkel I., Schweigert C., Correspondences of ribbon categories, Adv. Math. 199 (2006), 192-329, arXiv:math.CT/0309465.

[9] Fuchs J., Gannon T., Schaumann G., Schweigert C., The logarithmic Cardy case: boundary states and annuli, Nuclear Phys. B 930 (2018), 287-327, arXiv:1712.01922.

[10] Fuchs J., Runkel I., Schweigert C., TFT construction of RCFT correlators. I. Partition functions, Nuclear Phys. B 646 (2002), 353-497, arXiv:hep-th/0204148.

[11] Fuchs J., Runkel I., Schweigert C., TFT construction of RCFT correlators. II. Unoriented world sheets, Nuclear Phys. B 678 (2004), 511-637, arXiv:hep-th/0306164.

[12] Fuchs J., Runkel I., Schweigert C., TFT construction of RCFT correlators. III. Simple currents, Nuclear Phys. B 694 (2004), 277-353, arXiv:hep-th/0403157.

[13] Fuchs J., Runkel I., Schweigert C., TFT construction of RCFT correlators. IV. Structure constants and correlation functions, Nuclear Phys. B $\mathbf{7 1 5}$ (2005), 539-638, arXiv:hep-th/0412290.

[14] Fuchs J., Schweigert C., Consistent systems of correlators in non-semisimple conformal field theory, Adv. Math. 307 (2017), 598-639, arXiv:1604.01143.

[15] Goosen G., Oriented 123-TQFTs via string-nets and state-sums, Ph.D. Thesis, Stellenbosch University, 2018.

[16] Hatcher A., Lochak P., Schneps L., On the Teichmüller tower of mapping class groups, J. Reine Angew. Math. 521 (2000), 1-24.

[17] Kirillov Jr. A., String-net model of Turaev-Viro invariants, arXiv:1106.6033.

[18] Kirillov Jr. A., Balsam B., Turaev-Viro invariants as an extended TQFT, arXiv:1004.1533.

[19] Koenig R., Kuperberg G., Reichardt B.W., Quantum computation with Turaev-Viro codes, Ann. Physics 325 (2010), 2707-2749, arXiv:1002.2816.

[20] Kong L., Runkel I., Morita classes of algebras in modular tensor categories, Adv. Math. 219 (2008), 15481576, arXiv:0708.1897. 
[21] Kong L., Runkel I., Cardy algebras and sewing constraints. I, Comm. Math. Phys. 292 (2009), 871-912, arXiv:0807.3356.

[22] Levin M.A., Wen X.-G., String-net condensation: a physical mechanism for topological phases, Phys. Rev. B 71 (2005), 045110, 21 pages, arXiv:cond-mat/0404617.

[23] Ng S.-H., Schauenburg P., Higher Frobenius-Schur indicators for pivotal categories, in Hopf algebras and generalizations, Contemp. Math., Vol. 441, Amer. Math. Soc., Providence, RI, 2007, 63-90, arXiv:math.QA/0503167.

[24] Shimizu K., Non-degeneracy conditions for braided finite tensor categories, Adv. Math. 355 (2019), 106778 , 36 pages, arXiv:1602.06534.

[25] Traube M., Cardy algebras, sewing constraints and string-nets, arXiv:2009.11895.

[26] Turaev V., Virelizier A., On two approaches to 3-dimensional TQFTs, arXiv:1006.3501. 